\title{
Nonresistive dissipative magnetohydrodynamics from the Boltzmann equation in the 14-moment approximation
}

\section{Denicol, Gabriel S.}

2018-10-17

Denicol , G S , Huang , X-G, Molnár , E , Monteiro , G M , Niemi , H , Noronha , J , Rischke , D H \& Wang , Q 2018, ' Nonresistive dissipative magnetohydrodynamics from the Boltzmann equation in the 14-moment approximation ' , Physical Review D , vol. 98 , no. 7 , 076009 . https://doi.org/10.1103/PhysRevD.98.076009

http://hdl.handle.net/10138/258267

https://doi.org/10.1103/PhysRevD.98.076009

cc_by

publishedVersion

Downloaded from Helda, University of Helsinki institutional repository.

This is an electronic reprint of the original article.

This reprint may differ from the original in pagination and typographic detail.

Please cite the original version. 


\title{
Nonresistive dissipative magnetohydrodynamics from the Boltzmann equation in the 14-moment approximation
}

\author{
Gabriel S. Denicol, ${ }^{1}$ Xu-Guang Huang, ${ }^{2,3}$ Etele Molnár, ${ }^{4,5}$ Gustavo M. Monteiro, ${ }^{6}$ Harri Niemi, ${ }^{4,7,8}$ \\ Jorge Noronha, ${ }^{9}$ Dirk H. Rischke, ${ }^{4,10}$ and Qun Wang ${ }^{10}$ \\ ${ }^{1}$ Instituto de Física, Universidade Federal Fluminense, UFF, Niterói, 24210-346 RJ, Brazil \\ ${ }^{2}$ Physics Department and Center for Particle Physics and Field Theory, \\ Fudan University, Shanghai 200433, China \\ ${ }^{3}$ Key Laboratory of Nuclear Physics and Ion-beam Application (MOE), \\ Fudan University, Shanghai 200433, China \\ ${ }^{4}$ Institut für Theoretische Physik, Johann Wolfgang Goethe-Universität, \\ Max-von-Laue-Str. 1, D-60438 Frankfurt am Main, Germany \\ ${ }^{5}$ Institute of Physics and Technology, University of Bergen, Allegaten 55, 5007 Bergen, Norway \\ ${ }^{6}$ Instituto de Física Gleb Wataghin, Universidade Estadual de Campinas-UNICAMP, \\ 13083-859 Campinas, SP, Brazil \\ ${ }^{7}$ Department of Physics, University of Jyväskylä, P.O. Box 35, FI-40014 University of Jyväskylä, Finland \\ ${ }^{8}$ Helsinki Institute of Physics, P.O. Box 64, FI-00014 University of Helsinki, Finland \\ ${ }^{9}$ Instituto de Física, Universidade de São Paulo, Rua do Matão, 1371, Butantã, 05508-090, \\ São Paulo, SP, Brazil \\ ${ }^{10}$ Department of Modern Physics, University of Science and Technology of China, \\ Hefei, Anhui 230026, China
}

(Received 14 April 2018; published 17 October 2018)

\begin{abstract}
We derive the equations of motion of relativistic, nonresistive, second-order dissipative magnetohydrodynamics from the Boltzmann equation using the method of moments. We assume the fluid to be composed of a single type of point-like particles with vanishing dipole moment or spin, so that the fluid has vanishing magnetization and polarization. In a first approximation, we assume the fluid to be nonresistive, which allows to express the electric field in terms of the magnetic field. We derive equations of motion for the irreducible moments of the deviation of the single-particle distribution function from local thermodynamical equilibrium. We analyze the Navier-Stokes limit of these equations, reproducing previous results for the structure of the first-order transport coefficients. Finally, we truncate the system of equations for the irreducible moments using the 14-moment approximation, deriving the equations of motion of relativistic, nonresistive, second-order dissipative magnetohydrodynamics. We also give expressions for the new transport coefficients appearing due to the coupling of the magnetic field to the dissipative quantities.
\end{abstract}

DOI: 10.1103/PhysRevD.98.076009

\section{INTRODUCTION}

The success of relativistic fluid dynamics in describing the evolution of high-energy heavy-ion collisions [1] and the existence of very large magnetic fields in these collisions [2-5] has generated a lot of interest in observing the effects of the magnetic field on the fluid-dynamical evolution in these systems. The generic framework that couples the electromagnetic field to the dynamics of a fluid is referred to as magnetohydrodynamics $[6,7]$. There are several works where the effect of electromagnetic fields on the dynamics of heavy-ion collisions have been studied (for a review, see Ref. [8] and references therein), but so far they have been mostly based on the nonresistive, nondissipative formulation of relativistic magnetohydrodynamics. However, dissipation plays an important role in understanding the dynamics of heavy-ion collisions and in particular in explaining the magnitude of the observed collective flow (for a review, see Ref. [1] and references therein). Thus, it is essential to develop a relativistic formulation of dissipative magnetohydrodynamics.

In principle, the most simple dissipative fluid-dynamical theory is a relativistic generalization of Navier-Stokes theory, where the dissipative quantities, bulk viscous pressure, diffusion currents, and shear-stress tensor, are proportional to the gradients of the flow field and of thermodynamical quantities. In the absence of a magnetic field, the constants of proportionality are three scalar transport coefficients: the bulk viscosity, diffusion constant, and shear viscosity. A magnetic field breaks the isotropy of space, introducing several new transport coefficients [9-13], 
which assume different values in the direction of the magnetic field and in the direction orthogonal to it. The relativistic generalization of Navier-Stokes theory is, however, known to be acausal [14] and, at least, linearly unstable [15-17], rendering it ill-suited for practical use. Without the magnetic field, these problems were cured by the causal and stable "second-order" formalism of Israel and Stewart [18-20]. Israel-Stewart theory can be derived by starting from the relativistic Boltzmann equation employing the so-called 14-moment approximation [19,20], and the success of fluid dynamics in describing the dynamics of heavy-ion collisions is based on this formalism.

In this paper, we follow the same line of reasoning as Israel and Stewart, and derive a relativistic causal theory of second-order dissipative magnetohydrodynamics from the relativistic Boltzmann equation coupled to an electromagnetic field. As in the original formulation by Israel and Stewart, we restrict ourselves to a single-component system of spinless particles undergoing binary elastic collisions and use the 14-moment approximation in the framework developed in Refs. [21-23]. In a first step, we assume the fluid to have infinite electric conductivity (or zero resistivity), which allows to replace the electric field by the magnetic field and considerably simplifies the equations of motion. We remark that the assumption of infinite electric conductivity is an idealization which is hard (if not impossible) to realize in systems whose microscopic dynamics is described by the Boltzmann equation: the electric conductivity is a transport coefficient which is proportional to the mean free path of the particles, such as all other transport coefficients appearing in dissipative fluid dynamics, and thus should be of the same order of magnitude as the latter. Nevertheless, as nonresistive magnetohydrodynamics is a theory which finds widespread applications, we decided to first discuss the simpler case of a nonresistive (albeit dissipative) fluid. The generalization towards systems with finite conductivity will be addressed in a subsequent paper.

Let us add a few remarks on the length scales entering our discussion. (i) The Boltzmann equation is derived under the assumption that the collision term in this equation is local, implying that the mean free path $\lambda_{\text {mfp }}$ between collisions is much larger than the typical interaction length $\sqrt{\sigma / \pi}$, where $\sigma$ is the binary-collision cross section. (ii) The magnetic field leads to cyclotron motion of the charged particles. The curvature of the particle trajectories is given by the inverse Larmor radius $R_{L}^{-1}=\mathfrak{q} B / k_{\perp}$, where $\mathfrak{q}$ is the electric charge of the particles and $k_{\perp}$ is the momentum of the particle transverse to the direction of the magnetic induction field $\mathbf{B}$, which has magnitude $B=|\mathbf{B}|$ (in the following the magnetic induction field is in a simplifying, but somewhat incorrect, manner referred to as "magnetic field"). In our discussion we will assume that the magnetic field is sufficiently weak so that we can neglect the Landau quantization of the cyclotron motion. This implies that the thermal energy $\sim T$, where $T$ is the temperature, is much larger than the cyclotron frequency $\sim \sqrt{\mathfrak{q} B}$. In other words, the thermal wavelength $\beta_{0} \equiv$ $1 / T \ll R_{T}$, where $R_{T} \equiv\left(\mathfrak{q} B \beta_{0}\right)^{-1}$ is the Larmor radius of a particle with transverse momentum $k_{\perp}=T$. In the following, we refer to $R_{T}$ as the "thermal Larmor radius." Note that this condition does not necessarily imply that the magnetic field is weak in absolute magnitude; it only requires that the temperature of the system is sufficiently large, such that $T^{2} \gg \mathfrak{q} B$. While our discussion is valid when $\lambda_{\mathrm{mfp}} \gg \sqrt{\sigma / \pi}$ and $R_{T} \gg \beta_{0}$, there is a priori no constraint on the ratio $\xi_{B} \equiv \lambda_{\text {mfp }} / R_{T}=\mathfrak{q} B \beta_{0} \lambda_{\text {mfp }}$ [24], as long as the first two inequalities are fulfilled.

This paper is organized as follows. In Sec. II we review the structure of the equations of motion of magnetohydrodynamics, i.e., the evolution equations for energy and momentum coupled to Maxwell's equations for the electromagnetic fields. In Sec. III we present the magnetohydrodynamic equations of motion for the nonresistive, nondissipative fluid. In Sec. IV A we recall the method of moments and derive the equations of motion for the moments of the deviation of the single-particle distribution function from local thermodynamical equilibrium in the presence of a magnetic field. In Sec. IV B we show how the Navier-Stokes limit arises from the moment expansion. Finally, in Sec. IV C we derive the main result of this paper: the equations of motion for nonresistive, second-order dissipative magnetohydrodynamics. Section V concludes this work with a summary of the results and an outlook to future work.

We adopt natural Heaviside-Lorentz units, $\hbar=c=\epsilon_{0}=$ $\mu_{0}=k_{B}=1$. Our convention for the metric tensor is $g^{\mu \nu}=\operatorname{diag}(1,-1,-1,-1)$. The fluid four-velocity is $u^{\mu}(t, \mathbf{x})=\gamma(1, \mathbf{v})^{T}$, with $\gamma=\left(1-\mathbf{v}^{2}\right)^{-1 / 2}$, leading to the normalization $u^{\mu} u_{\mu} \equiv 1$. In the local rest (LR) frame of the fluid, $u_{L R}^{\mu}=(1, \mathbf{0})^{T}$. The four-momentum $k^{\mu}$ of particles is normalized to their rest mass $m_{0}, k^{\mu} k_{\mu}=m_{0}^{2}$. The rank-two projection operator onto the three-space orthogonal to $u^{\mu}$ is $\Delta^{\mu \nu}=g^{\mu \nu}-u^{\mu} u^{\nu}$. For a four-vector $A^{\mu}$, we define its projection onto the three-dimensional subspace orthogonal to $u^{\mu}$ as $A^{\langle\mu\rangle} \equiv \Delta_{\nu}^{\mu} A^{\nu}$. The rank-four projection operator is defined as $\Delta_{\alpha \beta}^{\mu \nu}=\frac{1}{2}\left(\Delta_{\alpha}^{\mu} \Delta_{\beta}^{\nu}+\Delta_{\beta}^{\mu} \Delta_{\alpha}^{\nu}\right)-\frac{1}{3} \Delta^{\mu \nu} \Delta_{\alpha \beta}$, which is symmetric and traceless. For a rank-two tensor, we define the symmetric, traceless projection onto the three-space orthogonal to $u^{\mu}$ as $A^{\langle\mu \nu\rangle} \equiv \Delta_{\alpha \beta}^{\mu \nu} A^{\alpha \beta}$. Our convention and useful relations for the rank-four Levi-Civita tensor $\epsilon^{\mu \nu \alpha \beta}$ are given in the Appendix.

\section{EQUATIONS OF MOTION OF MAGNETOHYDRODYNAMICS}

\section{A. Maxwell's equations and energy-momentum tensor of the electromagnetic field}

In a relativistically covariant formulation of electrodynamics, the electric field vector $\mathbf{E}$ and the magnetic field vector $\mathbf{B}$ constitute the components of the Faraday tensor 
$F^{\mu \nu}$. The latter is an antisymmetric (and hence traceless) rank-two tensor (and thus has six independent components, corresponding to the six components of $\mathbf{E}$ and $\mathbf{B}$ ). Without loss of generality it can be decomposed with respect to the fluid velocity as $[25,26]$

$$
F^{\mu \nu} \equiv E^{\mu} u^{\nu}-E^{\nu} u^{\mu}+\epsilon^{\mu \nu \alpha \beta} u_{\alpha} B_{\beta},
$$

while its Hodge dual is

$$
\tilde{F}^{\mu \nu} \equiv \frac{1}{2} \epsilon^{\mu \nu \alpha \beta} F_{\alpha \beta}=B^{\mu} u^{\nu}-B^{\nu} u^{\mu}-\epsilon^{\mu \nu \alpha \beta} u_{\alpha} E_{\beta} .
$$

Here we defined the electric field four-vector $E^{\mu} \equiv F^{\mu \nu} u_{\nu}$ and the magnetic field four-vector $B^{\mu} \equiv \tilde{F}^{\mu \nu} u_{\nu}=$ $\frac{1}{2} \epsilon^{\mu \nu \alpha \beta} F_{\alpha \beta} u_{\nu}$. Using the antisymmetry of the Faraday tensor and the rank-four Levi-Civita tensor, one readily realizes that $E^{\mu}$ and $B^{\mu}$ are orthogonal to the fluid velocity, $E^{\mu} u_{\mu}=0$ and $B^{\mu} u_{\mu}=0$. Moreover, in the local rest frame of the fluid, they coincide with the usual electric and magnetic fields, i.e., $E_{L R}^{\mu}=(0, \mathbf{E})^{T}$ and $B_{L R}^{\mu}=(0, \mathbf{B})^{T}$, with $\mathrm{E}^{i}=$ $F^{i 0}$ and $\mathrm{B}^{i}=-\frac{1}{2} \epsilon^{i j k} F_{j k}$. The electric field is a polar vector, while the magnetic field is an axial vector dual to $F_{j k}$.

The evolution of the electric and magnetic fields are given by Maxwell's equations,

$$
\begin{gathered}
\partial_{\mu} F^{\mu \nu}=\tilde{J}^{\nu}, \\
\partial_{\mu} \tilde{F}^{\mu \nu}=0,
\end{gathered}
$$

where the electric charge four-current $\mathfrak{J}^{\nu}$ serves as source for the electromagnetic field. It can be tensor-decomposed with respect to the fluid velocity [26,27],

$$
\mathfrak{J}^{\mu}=\mathbf{n} u^{\mu}+\mathfrak{V}^{\mu},
$$

where $\mathfrak{n}=u_{\mu} \tilde{\mathcal{J}}^{\mu}$ is the charge density in the local rest frame of the fluid and $\mathfrak{V}^{\mu} \equiv \Delta_{\nu}^{\mu} \mathfrak{S}^{\nu}$ is the charge diffusion four-current. The solution of Eqs. (3) and (4) determines the electromagnetic fields as functionals of $\mathfrak{J}^{\mu}$.

For nonpolarizable, nonmagnetizable fluids the electromagnetic stress-energy tensor is given by $[25,28]$

$$
T_{e m}^{\mu \nu}=-F^{\mu \lambda} F_{\lambda}^{\nu}+\frac{1}{4} g^{\mu \nu} F^{\alpha \beta} F_{\alpha \beta} .
$$

Using Maxwell's equations (3) and (4) one can show that

$$
\partial_{\mu} T_{e m}^{\mu \nu}=-F^{\nu \lambda} \tilde{\jmath}_{\lambda}
$$

\section{B. Particle four-current and energy-momentum tensor of the fluid}

For particles without a microscopic dipole moment or spin the canonical momentum coincides with the kinetic momentum [28]. Then, the particle four-current and energy-momentum tensor of the fluid are simply given by

$$
\begin{gathered}
N_{f}^{\mu} \equiv\left\langle k^{\mu}\right\rangle, \\
T_{f}^{\mu \nu} \equiv\left\langle k^{\mu} k^{\nu}\right\rangle .
\end{gathered}
$$

Here,

$$
\langle\cdots\rangle \equiv \int d K \cdots f_{\mathbf{k}},
$$

with $f_{\mathbf{k}}$ being the single-particle distribution function and $d K \equiv g d^{3} \mathbf{k} /\left[(2 \pi)^{3} k^{0}\right]$ being the Lorentz-invariant measure in momentum space, where $g$ is the degeneracy factor due to internal degrees of freedom (note, however, that the spin degeneracy is $2 J+1=1$, since we consider spin-zero particles), and $k^{0}=\sqrt{\mathbf{k}^{2}+m_{0}^{2}}$ is the on-shell energy.

The particle four-current and the energy-momentum tensor can be tensor-decomposed with respect to the fluid velocity,

$$
\begin{gathered}
N_{f}^{\mu}=n_{f} u^{\mu}+V_{f}^{\mu}, \\
T_{f}^{\mu \nu}=\varepsilon u^{\mu} u^{\nu}-P \Delta^{\mu \nu}+W^{\mu} u^{\nu}+W^{\nu} u^{\mu}+\pi^{\mu \nu},
\end{gathered}
$$

where the particle density $n_{f}$, the energy density $\varepsilon$, and the isotropic pressure $P$ are defined as

$$
\begin{gathered}
n_{f} \equiv N_{f}^{\mu} u_{\mu}=\left\langle E_{\mathbf{k}}\right\rangle, \\
\varepsilon \equiv T_{f}^{\mu \nu} u_{\mu} u_{\nu}=\left\langle E_{\mathbf{k}}^{2}\right\rangle, \\
P \equiv-\frac{1}{3} T_{f}^{\mu \nu} \Delta_{\mu \nu}=-\frac{1}{3}\left\langle\Delta^{\mu \nu} k_{\mu} k_{\nu}\right\rangle,
\end{gathered}
$$

with $E_{\mathbf{k}}=k^{\mu} u_{\mu}$ being the energy of a particle in the local rest frame of the fluid. The particle and energy-momentum diffusion currents orthogonal to the flow velocity are

$$
\begin{gathered}
V_{f}^{\mu} \equiv \Delta_{\nu}^{\mu} N_{f}^{\nu}=\left\langle k^{\langle\mu\rangle}\right\rangle, \\
W^{\mu} \equiv \Delta_{\alpha}^{\mu} T_{f}^{\alpha \beta} u_{\beta}=\left\langle E_{\mathbf{k}} k^{\langle\mu\rangle}\right\rangle,
\end{gathered}
$$

respectively, while the shear-stress tensor is

$$
\pi^{\mu \nu} \equiv \Delta_{\alpha \beta}^{\mu \nu} T_{f}^{\alpha \beta}=\left\langle k^{\langle\mu} k^{\nu\rangle}\right\rangle .
$$

For a single-component fluid, the electric charge and particle four-currents are related by

$$
\mathfrak{J}_{f}^{\mu} \equiv \mathfrak{q} N_{f}^{\mu}=\mathfrak{n}_{f} u^{\mu}+\mathfrak{V}_{f}^{\mu},
$$

where $\mathfrak{n}_{f} \equiv u_{\nu} \widetilde{\boldsymbol{J}}_{f}^{\nu} \equiv \mathfrak{q} u_{\nu} N_{f}^{\nu}=\mathfrak{q} n_{f}$ is the charge density in the local rest frame and $\mathfrak{V}_{f}^{\mu} \equiv \Delta_{\nu}^{\mu} \widetilde{J}_{f}^{\nu} \equiv \mathfrak{q} \Delta_{\nu}^{\mu} N_{f}^{\nu}=\mathfrak{q} V_{f}^{\mu}$ is 
the charge diffusion current. To leading order, the charge diffusion current is equal to the Ohmic induction current, $\mathfrak{q} V_{f}^{\mu} \simeq \widetilde{\mathcal{F}}_{\text {ind }}^{\mu}=\sigma_{E} E^{\mu}$.

The components of $\widetilde{J}_{f}^{\mu}$ and $T_{f}^{\mu \nu}$ contain 14 unknowns, or equivalently, for a given four-vector field $u^{\mu}$ the three scalar quantities $\mathfrak{n}_{f}, \varepsilon, P$, the two times three (equals six) independent components of $\mathfrak{V}_{f}^{\mu}$ and $W^{\mu}$, and the five independent components of $\pi^{\mu \nu}$. If the fluid velocity is a dynamical quantity, this would add another three unknowns (the three independent components of $u^{\mu}$ ). However, the fluid velocity can be chosen to be proportional to the charge fourcurrent, which eliminates the charge diffusion current $\mathfrak{V}_{f}^{\mu}$ (the so-called Eckart frame [29]), or to be proportional to the flow of energy, which eliminates the energy-momentum diffusion current $W^{\mu}$ (the so-called Landau frame [30]).

Let us assume that the only charge current in the system is that of the fluid, $\mathfrak{J}^{\mu} \equiv \mathfrak{J}_{f}^{\mu}$. If we project Maxwell's equation (3) onto $u_{\nu}$ and use Eqs. (1) and (5), we obtain,

$$
\nabla_{\mu} E^{\mu}+2 \omega_{\mu} B^{\mu}=\mathbf{n}_{f},
$$

where we introduced the three-space gradient $\nabla_{\mu} \equiv \Delta_{\mu}^{\alpha} \partial_{\alpha}$ and the vorticity four-vector

$$
\omega^{\mu}=\frac{1}{2} \epsilon^{\mu \nu \alpha \beta} u_{\nu} \partial_{\alpha} u_{\beta}
$$

In the following, we want to consider the nonresistive limit, i.e., the electric conductivity $\sigma_{E} \rightarrow \infty$. In this limit, the Ohmic conduction current $\widetilde{\jmath}_{\text {ind }}^{\mu}$ would diverge, unless we demand that $E^{\mu}=0$, or $\mathbf{E}=-\mathbf{v} \times \mathbf{B}$, so that $\mathfrak{J}_{\text {ind }}^{\mu} \simeq \mathfrak{V}_{f}^{\mu}$ remains finite. However, if $E^{\mu}=0$, we observe that the charge density of the fluid (and thus, for our singlecomponent system, the particle density of the fluid) assumes a value which is uniquely determined by the scalar product of the magnetic field four-vector and the fluid vorticity, $\mathbf{n}_{f}=\mathfrak{q} n_{f}=2 \omega_{\mu} B^{\mu},{ }^{1}$ and is no longer an independent variable. Projecting Eq. (3) with $\Delta_{\nu}^{\alpha}$, similar arguments apply to the charge diffusion current $\mathfrak{V}_{f}^{\nu}=\mathfrak{q} V_{f}^{\nu}$.

On the other hand, in dissipative fluid dynamics $n_{f}$ and $V_{f}^{\nu}$ are traditionally considered as four (out of 14) independent variables. In order to maintain this feature, we introduce an external current, $\tilde{\mathcal{J}}_{\mathrm{ext}}^{\mu}$, such that the total charge current (5) reads

$$
\widetilde{\jmath}^{\mu}=\widetilde{\mathfrak{J}}_{\mathrm{ext}}^{\mu}+\widetilde{\mathfrak{J}}_{f}^{\mu}
$$

\footnotetext{
${ }^{1}$ Amusingly, the corresponding term is of the same structure as the spin-vorticity coupling term discussed in Ref. [31], but the coefficient assumes a different value, since in that case it is determined by spin-1/2 fermions in the lowest Landau level, while here we deal with spinless particles and neglect Landau quantization.
}

Then, $n_{f}$ and $V_{f}^{\nu}$ become independent variables to be determined by the equations of motion for the fluid. In this case, our derivation of dissipative magnetohydrodynamics can be formulated in close analogy to the one of ordinary dissipative fluid dynamics for single-component systems. Note that the introduction of an external current does not affect our argument that $E^{\mu}$ must vanish in the limit of infinite conductivity.

\section{Equations of motion of magnetohydrodynamics}

The total energy-momentum tensor of the system is

$$
T^{\mu \nu}=T_{e m}^{\mu \nu}+T_{f}^{\mu \nu}
$$

Note that the separation of $T^{\mu \nu}$ into $T_{e m}^{\mu \nu}$ and $T_{f}^{\mu \nu}$ is not unique in the case of polarizable, magnetizable fluids [28]. This problem is absent here, as we consider a nonpolarizable, nonmagnetizable fluid.

While the charge current of the fluid is conserved,

$$
\partial_{\mu} \widetilde{\mathcal{J}}_{f}^{\mu}=0
$$

the total energy and momentum of the system are not, as the external charge current induces electromagnetic fields and thus feeds energy and momentum into the system. In analogy to Eq. (7) we have

$$
\partial_{\mu} T^{\mu \nu}=-F^{\nu \lambda} \tilde{\jmath}_{\mathrm{ext}, \lambda} .
$$

With Eq. (22), Eq. (7) reads

$$
\partial_{\mu} T_{e m}^{\mu \nu}=-F^{\nu \lambda}\left(\tilde{J}_{\mathrm{ext}, \lambda}+\tilde{J}_{f, \lambda}\right),
$$

and with Eq. (23) we can derive from Eq. (25) an equation of motion for the energy-momentum tensor of the fluid,

$$
\partial_{\mu} T_{f}^{\mu \nu}=F^{\nu \lambda} \tilde{\mho}_{f, \lambda} .
$$

Equations (24), (26), and (27) constitute the equations of motion of magnetohydrodynamics. While the energy and momentum of the electromagnetic fields change on account of the external charge current as well as the internal charge current of the particles in the fluid, Eq. (26), the energy and momentum of the fluid change only on account of the Lorentz force exerted on the charged particles within the fluid by the electromagnetic fields, Eq. (27). In general, neither energy and momentum of the electromagnetic fields nor that of the fluid are conserved separately. The total energy and momentum are only conserved in the absence of an external charge current, $\tilde{J}_{\text {ext }, \lambda}=0$, so that Eq. (25) becomes $\partial_{\mu} T^{\mu \nu}=0$. 


\section{NONRESISTIVE, NONDISSIPATIVE MAGNETOHYDRODYNAMICS}

\section{A. Assumption of nonresistivity}

The charge four-current induced by an electric field is $\widetilde{J}_{\text {ind }}^{\mu}=\sigma_{E} E^{\mu}$. A widely used approximation in applications of magnetohydrodynamics is the assumption that the fluid is nonresistive, i.e., ideally conducting, such that $\sigma_{E} \rightarrow \infty$. Then, as already stated, in order to have a finite induced charge current $\widetilde{F}_{\text {ind }}^{\mu}$ one has to demand $E^{\mu} \rightarrow 0$. From this condition it follows that, in an arbitrary frame, $\mathbf{E}=-\mathbf{v} \times \mathbf{B}$, such that the electric field can be eliminated from the equations of motion.

An ideally conducting fluid implies an infinite mean free path of charged particles, i.e., the free-streaming limit. However, in this paper we aim at deriving dissipative magnetohydrodynamics from an expansion around local thermodynamical equilibrium, which corresponds to the opposite limit of a vanishing mean free path. All transport coefficients appearing in the equations of motion are proportional to the mean free path of particles, which is assumed to be much smaller than the typical length scale over which fluid-dynamical quantities vary. In order to be consistent, the electric conductivity must be of the same order as the other transport coefficients (in fact, the famous Wiedemann-Franz law provides a unique relationship between the conductivity and the particle diffusion constant), and in principle we do not have the freedom to send it to infinity. In the case of a finite $\sigma_{E}$, we are in turn forced to consider a nonvanishing $E^{\mu}$. Nevertheless, since nonresistive magnetohydrodynamics is a theory which is widely applied to physical systems, we decided to separate the discussion by first treating the somewhat simpler case $E^{\mu}=0$ (corresponding to a nonresistive fluid), which is the subject of the present work, and then embarking on a treatment of the more complicated case $E^{\mu} \neq 0$, which will be the focus of a follow-up to this paper.

For $E^{\mu}=0$, the Faraday tensor (1) and its Hodge dual (2) simplify to

$$
\begin{gathered}
F^{\mu \nu} \rightarrow B^{\mu \nu}=\epsilon^{\mu \nu \alpha \beta} u_{\alpha} B_{\beta}, \\
\tilde{F}^{\mu \nu} \rightarrow \tilde{B}^{\mu \nu}=B^{\mu} u^{\nu}-B^{\nu} u^{\mu},
\end{gathered}
$$

while Maxwell's equations (3) and (4) reduce with Eq. (22) to

$$
\begin{gathered}
\epsilon^{\mu \nu \alpha \beta}\left(u_{\alpha} \partial_{\mu} B_{\beta}+B_{\beta} \partial_{\mu} u_{\alpha}\right)=\tilde{J}_{\mathrm{ext}}^{\nu}+\tilde{J}_{f}^{\nu}, \\
\dot{B}^{\mu}+B^{\mu} \theta=u^{\mu} \partial_{\nu} B^{\nu}+B^{\nu} \nabla_{\nu} u^{\mu},
\end{gathered}
$$

where $\dot{A} \equiv u^{\mu} \partial_{\mu} A$ is the comoving derivative of any quantity $A$ and $\theta \equiv \partial_{\mu} u^{\mu}$ is the expansion scalar.

The energy-momentum tensor of the electromagnetic field becomes

$$
T_{e m}^{\mu \nu} \rightarrow T_{B}^{\mu \nu}=\frac{B^{2}}{2}\left(u^{\mu} u^{\nu}-\Delta^{\mu \nu}-2 b^{\mu} b^{\nu}\right)
$$

where we introduced $B^{2} \equiv-B^{\mu} B_{\mu}$ and

$$
b^{\mu} \equiv \frac{B^{\mu}}{B},
$$

which is orthogonal to $u^{\mu}, b^{\mu} u_{\mu}=0$, and normalized to $b^{\mu} b_{\mu}=-1$.

For systems with a spatial anisotropy, as for instance induced by a magnetic field $[10,13,32,33]$, but not necessarily restricted to this case [34] (for a review see Ref. [35] and references therein), it is convenient to introduce a ranktwo operator projecting onto the two-dimensional subspace orthogonal to both $u^{\mu}$ and $b^{\mu}$,

$$
\Xi^{\mu \nu} \equiv g^{\mu \nu}-u^{\mu} u^{\nu}+b^{\mu} b^{\nu}=\Delta^{\mu \nu}+b^{\mu} b^{\nu} .
$$

Furthermore, since $B^{\mu \nu} B_{\mu \nu}=2 B^{2}$ it makes sense to introduce a new dimensionless antisymmetric tensor

$$
b^{\mu \nu} \equiv-\frac{B^{\mu \nu}}{B}=-\epsilon^{\mu \nu \alpha \beta} u_{\alpha} b_{\beta} .
$$

Obviously, $b^{\mu \nu} u_{\nu}=b^{\mu \nu} b_{\nu}=0$, while Eq. (A2) yields $b^{\mu \nu} b_{\mu \nu} \equiv-2 b^{\mu} b_{\mu}=2$. Moreover, with the help of Eq. (A1) one can show that

$$
b^{\mu \alpha} b_{\nu \alpha}=\Xi_{\nu}^{\mu}
$$

\section{B. Consequences for energy and momentum evolution of the fluid}

Already at this point we can draw conclusions from the assumption of nonresistivity for the equations of motion of magnetohydrodynamics. Projecting Eqs. (26) and (27) onto the direction of $u_{\nu}$ leads to

$$
\begin{gathered}
u_{\nu} \partial_{\mu} T_{B}^{\mu \nu}=B u_{\nu} b^{\nu \lambda}\left(\tilde{\jmath}_{\mathrm{ext}, \lambda}+\tilde{J}_{f, \lambda}\right)=0, \\
u_{\nu} \partial_{\mu} T_{f}^{\mu \nu}=-B u_{\nu} b^{\nu \lambda} \tilde{J}_{f, \lambda}=0,
\end{gathered}
$$

because of $u_{\nu} b^{\nu \lambda}=0$. The latter equation means that a magnetic field does not change the fluid energy, which is therefore separately conserved. This is easily understood since a magnetic field (contrary to an electric field) only changes the direction of the momenta of the particle, but not their energy. On the other hand, projecting Eqs. (26) and (27) onto the three-space orthogonal to $u_{\nu}$ we have

$$
\begin{aligned}
\Delta_{\nu}^{\alpha} \partial_{\mu} T_{B}^{\mu \nu} & =\left[B^{2} \dot{u}^{\alpha}-\nabla^{\alpha}\left(\frac{B^{2}}{2}\right)-\Delta_{\nu}^{\alpha} \partial_{\mu}\left(B^{2} b^{\mu} b^{\nu}\right)\right] \\
& =B b^{\alpha \lambda}\left(\mathfrak{V}_{\mathrm{ext}, \lambda}+\mathfrak{V}_{f, \lambda}\right),
\end{aligned}
$$




$$
\Delta_{\nu}^{\alpha} \partial_{\mu} T_{f}^{\mu \nu}=-B b^{\alpha \lambda} \mathfrak{Y}_{f, \lambda},
$$

where we employed Eq. (26) with Eq. (32) to obtain the first equation. For both equations we used the decomposition (5) [see also Eq. (19)], and employed the orthogonality $b^{\alpha \lambda} u_{\lambda}=0$.

The interpretation of Eq. (40) is that the momentum of the fluid changes on account of the interaction of the magnetic field with the charge diffusion current. Note that the magnetic field influences the dynamics of the fluid only by coupling to the dissipative part of the charge current. Without dissipation, the dynamics of the fluid is unaffected by the magnetic field; see Eq. (51) below.

\section{Equations of motion of nonresistive, nondissipative magnetohydrodynamics}

The equations of motion of nonresistive, nondissipative magnetohydrodynamics are obtained under the assumption that the fluid is in local thermodynamical equilibrium everywhere in space-time. In the case of dilute gases this assumption implies that the single-particle distribution function assumes the form [36]

$$
f_{\mathbf{k}} \rightarrow f_{0 \mathbf{k}}=\left[\exp \left(\beta_{0} E_{\mathbf{k}}-\alpha_{0}\right)+a\right]^{-1},
$$

with $\alpha_{0}=\mu \beta_{0}$, where $\mu$ is the chemical potential associated with the particle density $n_{0}$, and $a= \pm 1$ for fermions/ bosons, while $a \rightarrow 0$ for classical particles. Since we assumed that we can neglect the Landau quantization of single-particle energy eigenstates (see the Introduction), the distribution function is isotropic in the local frame, $E_{\mathbf{k}, L R}=\sqrt{\mathbf{k}^{2}+m_{0}^{2}}$. Local equilibrium means that the quantities $\alpha_{0}, \beta_{0}$, as well as the fluid velocity $u^{\mu}$ are functions of the space-time variable $x^{\mu}$. Since $f_{0 \mathbf{k}}$ depends solely on these five independent variables, and since $N_{f}^{\mu}$ and $T_{f}^{\mu \nu}$ computed from Eqs. (8) and (9) with $f_{0 \mathbf{k}}$ replacing $f_{\mathbf{k}}$ then also depend only on these five variables, the equations of motion of magnetohydrodynamics are closed.

In the following, we need the thermodynamic integrals

$$
I_{n q}\left(\alpha_{0}, \beta_{0}\right)=\frac{(-1)^{q}}{(2 q+1) ! !}\left\langle E_{\mathbf{k}}^{n-2 q}\left(\Delta^{\alpha \beta} k_{\alpha} k_{\beta}\right)^{q}\right\rangle_{0},
$$

where $\langle\cdots\rangle_{0} \equiv \int d K \cdots f_{0 \mathbf{k}}$ is defined in analogy to Eq. (10). Similarly, the auxiliary thermodynamic integrals are

$J_{n q} \equiv\left(\frac{\partial I_{n q}}{\partial \alpha_{0}}\right)_{\beta_{0}}=\frac{(-1)^{q}}{(2 q+1) ! !}\left\langle E_{\mathbf{k}}^{n-2 q}\left(\Delta^{\alpha \beta} k_{\alpha} k_{\beta}\right)^{q}\left(1-a f_{0 \mathbf{k}}\right)\right\rangle_{0}$.

Since $\left(\frac{\partial I_{n q}}{\partial \beta_{0}}\right)_{\alpha_{0}}=-J_{n+1, q}$, the total derivative is $d I_{n q}\left(\alpha_{0}, \beta_{0}\right) \equiv \frac{\partial I_{n q}}{\partial \alpha_{0}} d \alpha_{0}+\frac{\partial I_{n q}}{\partial \beta_{0}} d \beta_{0}=J_{n q} d \alpha_{0}-J_{n+1, q} d \beta_{0}$.

Using the equilibrium distribution function in Eqs. (8) and (9) we obtain the conserved quantities in the form for a nondissipative fluid,

$$
\begin{gathered}
N_{f 0}^{\mu} \equiv\left\langle k^{\mu}\right\rangle_{0}=n_{f 0} u^{\mu}, \\
T_{f 0}^{\mu \nu} \equiv\left\langle k^{\mu} k^{\nu}\right\rangle_{0}=\varepsilon_{0} u^{\mu} u^{\nu}-P_{0} \Delta^{\mu \nu},
\end{gathered}
$$

where

$$
\begin{gathered}
n_{f 0} \equiv N_{f 0}^{\mu} u_{\mu}=I_{10}, \\
\varepsilon_{0} \equiv T_{f 0}^{\mu \nu} u_{\mu} u_{\nu}=I_{20}, \\
P_{0} \equiv-\frac{1}{3} T_{f 0}^{\mu \nu} \Delta_{\mu \nu}=I_{21} .
\end{gathered}
$$

Therefore, the total energy-momentum tensor of a nonresistive, nondissipative fluid reads

$$
\begin{aligned}
T_{f 0+B}^{\mu \nu} & \equiv T_{f 0}^{\mu \nu}+T_{B}^{\mu \nu} \\
& =\left(\varepsilon_{0}+\frac{B^{2}}{2}\right) u^{\mu} u^{\nu}-\left(P_{0}+\frac{B^{2}}{2}\right) \Delta^{\mu \nu}-B^{2} b^{\mu} b^{\nu} .
\end{aligned}
$$

An immediate consequence of the assumptions of nonresistivity as well as nondissipativity is that the energy and momentum of the fluid are separately conserved,

$$
\partial_{\mu} T_{f 0}^{\mu \nu}=0 .
$$

This follows immediately from Eq. (27), since $F^{\nu \lambda} \tilde{\jmath}_{\lambda} \rightarrow$ $-B b^{\nu \lambda} \mathbf{n} u_{\lambda}=0$, but it also follows from Eqs. (38) and (40), since $\mathfrak{V}_{f, \lambda} \equiv 0$ for a nondissipative fluid. The energy of the magnetic field is conserved on account of Eq. (37), but the momentum is only conserved when $\mathfrak{V}_{\mathrm{ext}, \lambda}=0$, cf. Eq. (39).

\section{NONRESISTIVE, DISSIPATIVE MAGNETOHYDRODYNAMICS}

In this section, we derive the equations of motion of nonresistive, dissipative magnetohydrodynamics for a fluid consisting of a single type of point-like particles without dipole moment or spin. We also assume that the particles undergo binary elastic collisions only. Starting from the Boltzmann equation in the presence of an external electromagnetic field, we first derive the (infinite) 
set of equations of motion for the irreducible moments of the deviation

$$
\delta f_{\mathbf{k}} \equiv f_{\mathbf{k}}-f_{0 \mathbf{k}}
$$

of the single-particle distribution function from isotropic local thermodynamical equilibrium. Then we truncate this set using the 14-moment approximation. Our treatment follows closely that of Refs. [22,23], extending the latter by terms arising from the magnetic field. Note that our assumption $\beta_{0} \ll R_{T}$ (see the Introduction) allows us to neglect Landau quantization; otherwise $f_{0 \mathbf{k}}$ would be anisotropic. In principle, however, this case can be discussed using the formalism presented in Ref. [34]. An anisotropy also emerges when using a $f_{0 \mathbf{k}}$ which is a solution of the Vlasov equation [32,33], or an anisotropic distribution function parametrizing deviations from local equilibrium [37].

\section{A. Equations of motion for the irreducible moments}

The relativistic Boltzmann equation coupled to an electromagnetic field $[25,38]$ is

$$
k^{\mu} \partial_{\mu} f_{\mathbf{k}}+\mathfrak{q} F^{\mu \nu} k_{\nu} \frac{\partial}{\partial k^{\mu}} f_{\mathbf{k}}=C[f] .
$$

Here the assumption is that the electromagnetic field $F^{\mu \nu}$ changes the momenta $k^{\mu}$ of particles carrying charge $\mathfrak{q}$ on large space-time scales $\sim R_{T}$, while the collision term, being a quantity which is local in space-time, redistributes them on small space-time scales $\sim \sqrt{\sigma / \pi}$. We remark that if the particles carry a dipole moment or spin, there would be an additional term on the left-hand side [28]. Note that for Eq. (53) it does not matter whether the electromagnetic field is generated exclusively via the charge current of the particles, $\tilde{J}_{f}^{\nu}$ as source term in the inhomogeneous Maxwell equations, or exclusively via an external charge current $\tilde{J}_{\text {ext }}^{\nu}$, or by a combination of both. However, on account of our remarks made at the end of Sec. II B, only the case of a nonvanishing external charge current allows to treat the particle current $N_{f}^{\mu}$ as an independent fluid-dynamical variable.

Under the assumption that the particles undergo binary elastic collisions only, the collision term reads

$$
\begin{aligned}
C[f]= & \frac{1}{2} \int d K^{\prime} d P d P^{\prime}\left[W_{\mathbf{p p}^{\prime} \rightarrow \mathbf{k} \mathbf{k}^{\prime}} f_{\mathbf{p}} f_{\mathbf{p}^{\prime}}\left(1-a f_{\mathbf{k}}\right)\left(1-a f_{\mathbf{k}^{\prime}}\right)\right. \\
& \left.-W_{\mathbf{k k}^{\prime} \rightarrow \mathbf{p} \mathbf{p}^{\prime}} f_{\mathbf{k}} f_{\mathbf{k}^{\prime}}\left(1-a f_{\mathbf{p}}\right)\left(1-a f_{\mathbf{p}^{\prime}}\right)\right]
\end{aligned}
$$

where the factors $1-a f$ represent the corrections from quantum statistics. The invariant transition rate $W_{\mathbf{k} \mathbf{k}^{\prime} \rightarrow \mathbf{p p}^{\prime}}$ satisfies detailed balance, $W_{\mathbf{k k}^{\prime} \rightarrow \mathbf{p p}^{\prime}}=W_{\mathbf{p p}^{\prime} \rightarrow \mathbf{k k}^{\prime}}$, and is symmetric with respect to the exchange of momenta, $W_{\mathbf{k k}^{\prime} \rightarrow \mathbf{p} \mathbf{p}^{\prime}}=W_{\mathbf{k}^{\prime} \mathbf{k} \rightarrow \mathbf{p} \mathbf{p}^{\prime}}=W_{\mathbf{k k}^{\prime} \rightarrow \mathbf{p}^{\prime} \mathbf{p}}$.
Following Refs. [22,23] we define the irreducible moments of $\delta f_{\mathbf{k}}$ as ${ }^{2}$

$$
\rho_{r}^{\mu_{1} \cdots \mu_{n}} \equiv\left\langle E_{\mathbf{k}}^{r} k^{\left\langle\mu_{1}\right.} \cdots k^{\left.\mu_{n}\right\rangle}\right\rangle_{\delta}
$$

where $\langle\cdots\rangle_{\delta}=\int d K \cdots \delta f_{\mathbf{k}}$. Here, the irreducible tensor of rank $\ell$ is defined as

$$
k^{\left\langle\mu_{1}\right.} \cdots k^{\left.\mu_{\ell}\right\rangle}=\Delta_{\nu_{1} \cdots \nu_{\ell}}^{\mu_{1} \cdots \mu_{\ell}} k^{\nu_{1}} \cdots k^{\nu_{\ell}},
$$

where the rank- $2 \ell$ symmetric and traceless projection tensor $\Delta_{\nu_{1} \cdots \nu_{\ell}}^{\mu_{1} \cdots \mu_{\ell}}$ is a straightforward generalization of the rank-four projection tensor $\Delta_{\alpha \beta}^{\mu \nu}$ introduced above (for more details on how to construct the former, see Refs. [34,38]). The irreducible tensors $1, k^{\langle\mu\rangle}, k^{\langle\mu} k^{\nu\rangle}, k^{\langle\mu} k^{\nu} k^{\lambda\rangle}, \ldots$ form a complete basis in momentum space and satisfy the following orthogonality condition:

$$
\begin{aligned}
& \int d K \mathrm{~F}\left(E_{\mathbf{k}}\right) k^{\left\langle\mu_{1}\right.} \cdots k^{\left.\mu_{\ell}\right\rangle} k_{\left\langle\nu_{1}\right.} \cdots k_{\left.\nu_{n}\right\rangle} \\
& =\frac{\ell ! \delta_{\ell n}}{(2 \ell+1) ! !} \Delta_{\nu_{1} \cdots \nu_{\ell}}^{\mu_{1} \cdots \mu_{\ell}} \int d K \mathrm{~F}\left(E_{\mathbf{k}}\right)\left(\Delta^{\alpha \beta} k_{\alpha} k_{\beta}\right)^{\ell},
\end{aligned}
$$

where $\mathrm{F}\left(E_{\mathbf{k}}\right)$ is a sufficiently rapidly converging (but otherwise arbitrary) function of $E_{\mathbf{k}}$.

The deviations of the particle four-current and the fluid energy-momentum tensor from their local equilibrium values $N_{f 0}^{\mu}, T_{f 0}^{\mu \nu}$ are

$$
\begin{gathered}
\delta N_{f}^{\mu} \equiv\left\langle k^{\mu}\right\rangle_{\delta}=\delta n_{f} u^{\mu}+V_{f}^{\mu}, \\
\delta T_{f}^{\mu \nu} \equiv\left\langle k^{\mu} k^{\nu}\right\rangle_{\delta}=\delta \varepsilon u^{\mu} u^{\nu}-\Pi \Delta^{\mu \nu}+W^{\mu} u^{\nu}+W^{\nu} u^{\mu}+\pi^{\mu \nu},
\end{gathered}
$$

where the corrections to particle density, energy density, and isotropic pressure are

$$
\begin{gathered}
\delta n_{f} \equiv \delta N_{f}^{\mu} u_{\mu}=\rho_{1}, \\
\delta \varepsilon \equiv \delta T_{f}^{\mu \nu} u_{\mu} u_{\nu}=\rho_{2}, \\
\Pi \equiv-\frac{1}{3} \delta T_{f}^{\mu \nu} \Delta_{\mu \nu}=-\frac{m_{0}^{2}}{3} \rho_{0}+\frac{\rho_{2}}{3} .
\end{gathered}
$$

\footnotetext{
${ }^{2} \mathrm{~A}$ tensor is called irreducible when it is irreducible under a group $G$ consisting of Lorentz transformations that leave $u^{\mu}$ invariant. Let $F$ be a subgroup of $G$ consisting of Lorentz transformations that leave both $u^{\mu}$ and $b^{\mu}$ invariant. An irreducible tensor under $G$ may be reducible under $F$. This reduction of symmetry leads to a larger number of transport coefficients in dissipative magnetohydrodynamics than in ordinary dissipative fluid dynamics; see Sec. IV B.
} 
The particle and energy-momentum diffusion currents orthogonal to the fluid velocity are

$$
\begin{gathered}
V_{f}^{\mu} \equiv \Delta_{\nu}^{\mu} \delta N_{f}^{\nu}=\rho_{0}^{\mu}, \\
W^{\mu} \equiv \Delta_{\alpha}^{\mu} \delta T_{f}^{\alpha \beta} u_{\beta}=\rho_{1}^{\mu},
\end{gathered}
$$

while the shear-stress tensor is

$$
\pi^{\mu \nu} \equiv \Delta_{\alpha \beta}^{\mu \nu} \delta T_{f}^{\alpha \beta}=\rho_{0}^{\mu \nu}
$$

Choosing the Landau frame [30] to determine the fluid velocity implies

$$
u^{\mu}=\frac{T_{f}^{\mu \nu} u_{\nu}}{\sqrt{u_{\alpha} T_{f}^{\alpha \beta} T_{f, \beta \gamma} u^{\gamma}}}, \quad \rho_{1}^{\mu}=0 .
$$

The parameters $\alpha_{0}$ and $\beta_{0}$ entering $f_{0 \mathbf{k}}$ are determined by the so-called Landau matching conditions, i.e., demanding that the particle density and energy density resulting from $f_{\mathbf{k}}$ are identical with those resulting from $f_{0 \mathbf{k}}$, $n_{f}=n_{f 0}, \varepsilon=\varepsilon_{0}$, or in other words

$$
\delta n_{f}=\rho_{1}=0, \quad \delta \varepsilon=\rho_{2}=0 .
$$

Then, the charge four-current and total energymomentum tensor in nonresistive, dissipative magnetohydrodynamics are

$$
\widetilde{\mathfrak{J}}_{f}^{\mu} \equiv \mathfrak{n}_{f} u^{\mu}+\mathfrak{\mathfrak { V }}_{f}^{\mu}
$$

$$
\begin{aligned}
T^{\mu \nu} \equiv & T_{f}^{\mu \nu}+T_{B}^{\mu \nu} \\
= & \left(\varepsilon_{0}+\frac{B^{2}}{2}\right) u^{\mu} u^{\nu}-\left(P_{0}+\Pi+\frac{B^{2}}{2}\right) \Delta^{\mu \nu} \\
& -B^{2} b^{\mu} b^{\nu}+\pi^{\mu \nu} .
\end{aligned}
$$

Equations (24) and (27) with Eqs. (68) and (69) together with the thermodynamical identities (42) and (43) lead to the following equations of motion for $\alpha_{0}, \beta_{0}$, and $u^{\mu}$ :

$$
\begin{aligned}
\dot{\alpha}_{0}= & \frac{1}{D_{20}}\left[-J_{30}\left(n_{f 0} \theta+\partial_{\mu} V_{f}^{\mu}\right)+J_{20}\left(\varepsilon_{0}+P_{0}+\Pi\right) \theta\right. \\
& \left.-J_{20} \pi^{\mu \nu} \sigma_{\mu \nu}\right], \\
\dot{\beta}_{0}= & \frac{1}{D_{20}}\left[-J_{20}\left(n_{f 0} \theta+\partial_{\mu} V_{f}^{\mu}\right)+J_{10}\left(\varepsilon_{0}+P_{0}+\Pi\right) \theta\right. \\
& \left.-J_{10} \pi^{\mu \nu} \sigma_{\mu \nu}\right],
\end{aligned}
$$

and

$$
\begin{aligned}
\dot{u}^{\mu}= & \frac{1}{\varepsilon_{0}+P_{0}}\left[\frac{n_{f 0}}{\beta_{0}}\left(\nabla^{\mu} \alpha_{0}-h_{0} \nabla^{\mu} \beta_{0}\right)-\Delta_{\nu}^{\mu} \partial_{\kappa} \pi^{\kappa \nu}\right. \\
& \left.-\Pi \dot{u}^{\mu}+\nabla^{\mu} \Pi-\mathfrak{q} B b^{\mu \nu} V_{f, \nu}\right],
\end{aligned}
$$

where $D_{n q} \equiv J_{n+1, q} J_{n-1, q}-J_{n q}^{2}, h_{0} \equiv\left(\varepsilon_{0}+P_{0}\right) / n_{f 0}$ is the enthalpy per particle, and $\sigma^{\mu \nu}=\nabla^{\langle\mu} u^{\nu\rangle}$ is the shear tensor. The equations of motion for $\alpha_{0}$ and $\beta_{0}$ are the same as Eqs. (39)-(40) of Ref. [22]; however Eq. (72) contains an additional term due to the magnetic field when compared to Eq. (41) of Ref. [22].

We now use Eq. (52) to replace $f_{\mathbf{k}}$ by $\delta f_{\mathbf{k}}$ in the Boltzmann equation (53). Then, we take moments of the Boltzmann equation (53) in momentum space. With the definitions

$$
\dot{\rho}_{r}^{\left\langle\mu_{1} \cdots \mu_{\ell}\right\rangle} \equiv \Delta_{\nu_{1} \cdots \nu_{\ell}}^{\mu_{1} \cdots \mu_{\ell}} u^{\alpha} \partial_{\alpha} \rho_{r}^{\nu_{1} \cdots \nu_{\ell}},
$$

and

$$
\mathcal{C}_{r}^{\left\langle\mu_{1} \cdots \mu_{\ell}\right\rangle} \equiv \Delta_{\nu_{1} \cdots \nu_{\ell}}^{\mu_{1} \cdots \mu_{\ell}} \int d K E_{\mathbf{k}}^{r} k^{\nu_{1}} \cdots k^{\nu_{\ell}} C[f]
$$

we obtain the equations of motion for the irreducible moments, similarly as shown in Refs. [22,23].

The equation of motion for the irreducible tensors of rank zero reads

$$
\begin{aligned}
\dot{\rho}_{r}-C_{r-1}= & \alpha_{r}^{(0)} \theta+\frac{G_{3 r}}{D_{20}} \partial_{\mu} V_{f}^{\mu}+\frac{\theta}{3}\left[m_{0}^{2}(r-1) \rho_{r-2}\right. \\
& \left.-(r+2) \rho_{r}-3 \frac{G_{2 r}}{D_{20}} \Pi\right] \\
& +r \rho_{r-1}^{\mu} \dot{u}_{\mu}-\nabla_{\mu} \rho_{r-1}^{\mu} \\
& +\left[(r-1) \rho_{r-2}^{\mu \nu}+\frac{G_{2 r}}{D_{20}} \pi^{\mu \nu}\right] \sigma_{\mu \nu},
\end{aligned}
$$

where we have defined $G_{n m}=J_{n, 0} J_{m, 0}-J_{n-1,0} J_{m+1,0}$. Note that the contribution of the magnetic field vanishes for any scalar moment and exactly corresponds to Eq. (35) of Ref. [22]. However, the magnetic field is still present and affects the fluid motion through the acceleration equation, Eq. (72), as well as through the equations of motion for the irreducible moments of rank higher than zero (see below).

The equation of motion for the irreducible tensors of rank one is 


$$
\begin{aligned}
\dot{\rho}_{r}^{\langle\mu\rangle}-C_{r-1}^{\langle\mu\rangle}= & \alpha_{r}^{(1)} \nabla^{\mu} \alpha_{0}+r \rho_{r-1}^{\mu \nu} \dot{u}_{\nu}-\frac{1}{3} \nabla^{\mu}\left[m_{0}^{2} \rho_{r-1}-\rho_{r+1}\right]-\Delta_{\alpha}^{\mu}\left(\nabla_{\nu} \rho_{r-1}^{\alpha \nu}+\alpha_{r}^{h} \partial_{\kappa} \pi^{\kappa \alpha}\right) \\
& +\frac{1}{3}\left[m_{0}^{2}(r-1) \rho_{r-2}^{\mu}-(r+3) \rho_{r}^{\mu}\right] \theta+(r-1) \rho_{r-2}^{\mu \nu \lambda} \sigma_{\mu \nu}+\frac{1}{5} \sigma^{\mu \nu}\left[m_{0}^{2}(2 r-2) \rho_{r-2, \nu}-(2 r+3) \rho_{r, \nu}\right]+\rho_{r, \nu} \omega^{\mu \nu} \\
& +\frac{1}{3}\left[m_{0}^{2} r \rho_{r-1}-(r+3) \rho_{r+1}-3 \alpha_{r}^{h} \Pi\right] \dot{u}^{\mu}+\alpha_{r}^{h} \nabla^{\mu} \Pi-\alpha_{r}^{h} \mathfrak{q} B b^{\mu \nu} V_{f, \nu}-\mathfrak{q} B b^{\mu \nu} \rho_{r-1, \nu},
\end{aligned}
$$

where $\omega^{\mu \nu}=\left(\nabla^{\mu} u^{\nu}-\nabla^{\nu} u^{\mu}\right) / 2$ is the vorticity tensor. The two terms in the last line are new as compared to Eq. (36) of Ref. [22] and explicitly contain the magnetic field.

The equation of motion for the irreducible moments of tensor of rank two is

$$
\begin{aligned}
\dot{\rho}_{r}^{\langle\mu \nu\rangle}-C_{r-1}^{\langle\mu \nu\rangle}= & 2 \alpha_{r}^{(2)} \sigma^{\mu \nu}+\frac{2}{15}\left[m_{0}^{4}(r-1) \rho_{r-2}-(2 r+3) m_{0}^{2} \rho_{r}+(r+4) \rho_{r+2}\right] \sigma^{\mu \nu}+\frac{2}{5} \dot{u}^{\langle\mu}\left[m_{0}^{2} r \rho_{r-1}^{\nu\rangle}-(r+5) \rho_{r+1}^{\nu\rangle}\right] \\
& -\frac{2}{5}\left[\nabla^{\langle\mu}\left(m_{0}^{2} \rho_{r-1}^{\nu\rangle}-\rho_{r+1}^{\nu\rangle}\right)\right]+r \rho_{r-1}^{\mu \nu \gamma} \dot{u}_{\gamma}-\Delta_{\alpha \beta}^{\mu \nu} \nabla_{\lambda} \rho_{r-1}^{\alpha \beta \lambda}+(r-1) \rho_{r-2}^{\mu \nu \lambda} \sigma_{\lambda \kappa}+2 \rho_{r}^{\lambda\langle\mu} \omega_{\lambda}^{\nu\rangle}+\frac{1}{3}\left[m_{0}^{2}(r-1) \rho_{r-2}^{\mu \nu}\right. \\
& \left.-(r+4) \rho_{r}^{\mu \nu}\right] \theta+\frac{2}{7}\left[m_{0}^{2}(2 r-2) \rho_{r-2}^{\kappa\langle\mu}-(2 r+5) \rho_{r}^{\kappa\langle\mu}\right] \sigma_{\kappa}^{\nu\rangle}-2 \mathfrak{q} B b^{\alpha \beta} \Delta_{\alpha \kappa}^{\mu \nu} g_{\lambda \beta} \rho_{r-1}^{\kappa \lambda},
\end{aligned}
$$

where only the last term is new when compared to Eq. (37) of Ref. [22] and explicitly contains the magnetic field. Here we also defined the following coefficients which are formally unchanged from Eqs. (42)-(44) of Ref. [22]:

$$
\begin{gathered}
\alpha_{r}^{(0)}=(1-r) I_{r 1}-I_{r 0}-\frac{n_{f 0}}{D_{20}}\left(h_{0} G_{2 r}-G_{3 r}\right), \\
\alpha_{r}^{(1)}=J_{r+1,1}-h_{0}^{-1} J_{r+2,1}, \\
\alpha_{r}^{(2)}=I_{r+2,1}+(r-1) I_{r+2,2}, \\
\alpha_{r}^{h}=-\frac{\beta_{0}}{\varepsilon_{0}+P_{0}} J_{r+2,1} .
\end{gathered}
$$

The collision integral can be linearized using Eq. (52) and written as

$$
C_{r-1}^{\left\langle\mu_{1} \cdots \mu_{\ell}\right\rangle} \equiv-\sum_{n=0}^{N_{\ell}} \mathcal{A}_{r n}^{(\ell)} \rho_{n}^{\mu_{1} \cdots \mu_{\ell}}
$$

where the coefficient $\mathcal{A}_{r n}^{(\ell)}$ contains time scales $\sim \lambda_{\text {mfp }}$. In order to obtain this result, we have assumed that the magnetic field does not modify the collision integral, so that we were able to employ the orthogonality relation (57); for details see Ref. [22].

Note that, once the equations of motion (75)-(77) (and in principle those for all higher-rank tensors) are solved and the complete set of irreducible moments is determined, one can reconstruct the single-particle distribution $f_{\mathbf{k}}$ as a solution of the Boltzmann equation. Following Refs. [22,23],

$$
f_{\mathbf{k}}=f_{0 \mathbf{k}}+f_{0 \mathbf{k}}\left(1-a f_{0 \mathbf{k}}\right) \sum_{\ell=0}^{\infty} \sum_{n=0}^{N_{\ell}} \rho_{n}^{\mu_{1} \cdots \mu_{\ell}} k_{\left\langle\mu_{1}\right.} \cdots k_{\left.\mu_{\ell}\right\rangle} \mathcal{H}_{\mathbf{k} n}^{(\ell)} .
$$

We remark that this relation is an exact equality (i.e., $f_{\mathbf{k}}$ is an exact solution of the Boltzmann equation) only if we take $N_{\ell} \rightarrow \infty$. In practice, however, one has to truncate the sum over $n$ at some finite value, $N_{\ell}<\infty$. The same holds for the sum over $\ell$. Since there are no tensors of rank higher than two in fluid dynamics, this sum is usually restricted to $\ell \leq 2$. Furthermore, this also implies that higher-rank tensors on the right-hand sides of the equations of motion (75)-(77) will be subsequently neglected.

The coefficients $\mathcal{H}_{\mathbf{k} n}^{(\ell)}$ are defined as

$$
\mathcal{H}_{\mathbf{k} n}^{(\ell)}=\frac{(-1)^{\ell}}{\ell ! J_{2 \ell, \ell}} \sum_{i=n}^{N_{\ell}} \sum_{m=0}^{i} a_{\mathrm{in}}^{(\ell)} a_{i m}^{(\ell)} E_{\mathbf{k}}^{m}
$$

where the coefficients $a_{i j}^{(\ell)}$ can be written in terms of thermodynamic integrals and are calculated via GramSchmidt orthogonalization; for details see Ref. [22].

In preparation for a suitable truncation of the infinite set of equations of motion for the irreducible moments, we note that an irreducible moment of arbitrary order $r$ and tensor rank $\ell$ can always be expressed as a linear combination of irreducible moments of all orders $n$ and the same tensor rank, 


$$
\begin{aligned}
\rho_{r}^{\mu_{1} \cdots \mu_{\ell}}= & \sum_{n=0}^{N_{\ell}} \rho_{n}^{\mu_{1} \cdots \mu_{\ell}} \mathcal{F}_{-r, n}^{(\ell)}=\sum_{n=0}^{N_{\ell}} \rho_{n}^{\mu_{1} \cdots \mu_{\ell}} \\
& \times \sum_{i=n}^{N_{\ell}} \sum_{m=0}^{i} a_{\text {in }}^{(\ell)} a_{i m}^{(\ell)} \frac{J_{r+m+2 \ell, \ell}}{J_{2 \ell, \ell}},
\end{aligned}
$$

where

$\mathcal{F}_{r n}^{(\ell)}=\frac{\ell !}{(2 \ell+1) ! !} \int d K E_{\mathbf{k}}^{-r} \mathcal{H}_{\mathbf{k} n}^{(\ell)}\left(\Delta^{\alpha \beta} k_{\alpha} k_{\beta}\right)^{\ell} f_{0 \mathbf{k}}\left(1-a f_{0 \mathbf{k}}\right)$.

The first equality of Eq. (85) is proven using the orthogonality (57) of the irreducible moments and their definition (55). The second equality of Eq. (85) is shown using the definitions of the auxiliary thermodynamic integrals (43) and of the coefficients (84). Note that Eq. (85) is an identity for $0 \leq r \leq N_{\ell}$, while it is an approximation for $r$ outside this range, unless $N_{\ell} \rightarrow \infty$. The accuracy of this approximation can be systematically improved by increasing $N_{\ell}$. In the remainder of this paper, however, we will restrict ourselves to the so-called 14-moment approximation, i.e., we will assume $N_{0}=2, N_{1}=1$, and $N_{2}=0$ [22].

\section{B. The Navier-Stokes approximation}

Besides a suitable truncation of Eqs. (75)-(77), we also need a scheme to power count the various terms in these equations, in order to define the order of the approximation we are considering. We assume that quantities representing deviations from local thermodynamical equilibrium, like the irreducible moments, are of first order in some small parameter. Furthermore, since macroscopic fields like $\alpha_{0}\left(x^{\mu}\right), \beta_{0}\left(x^{\mu}\right)$, and $u^{\mu}\left(x^{\mu}\right)$ vary on space-time scales that are much larger than the microscopic scales contained in the collision integral, we also assume that derivatives of these fields are of first order in that small parameter.

In the Navier-Stokes approximation, all second-order terms, i.e., terms involving products of irreducible moments and derivatives of $\alpha_{0}, \beta_{0}$, and $u^{\mu}$, or derivatives of irreducible moments are neglected, leaving only the collision integrals [in linearized form; see Eq. (82)] on the left-hand sides and the first terms as well as the last terms involving the magnetic field on the right-hand sides of Eqs. (75)-(77). Bringing the latter ones to the left-hand side results in the following set of equations:

$$
\begin{gathered}
\sum_{n=0, \neq 1,2}^{N_{0}} \mathcal{A}_{r n}^{(0)} \rho_{n}=\alpha_{r}^{(0)} \theta, \\
\sum_{n=0, \neq 1}^{N_{1}}\left[\mathcal{A}_{r n}^{(1)} g^{\mu \nu}+\mathfrak{q} B\left(\mathcal{F}_{1-r, n}^{(1)}+\alpha_{r}^{h} \delta_{n 0}\right) b^{\mu \nu}\right] \rho_{n, \nu}=\alpha_{r}^{(1)} \nabla^{\mu} \alpha_{0},
\end{gathered}
$$

$$
\sum_{n=0}^{N_{2}}\left[\mathcal{A}_{r n}^{(2)} g_{\alpha}^{\mu} g_{\beta}^{\nu}+\mathfrak{q} B \mathcal{F}_{1-r, n}^{(2)}\left(b_{\beta}^{\mu} g_{\alpha}^{\nu}+b_{\beta}^{\nu} g_{\alpha}^{\mu}\right)\right] \rho_{n}^{\alpha \beta}=2 \alpha_{r}^{(2)} \sigma^{\mu \nu}
$$

In physical terms, it is assumed that the irreducible moments no longer evolve in time and assume their asymptotic solution given solely by the first-order terms on the right-hand side, multiplied by the inverse of the coefficient matrix on the left-hand side. The formal solution of this set of equations is

$$
\begin{gathered}
\rho_{r}=\zeta_{r}^{\mu \nu} \partial_{\mu} u_{\nu}, \\
\rho_{r}^{\mu}=\kappa_{r}^{\mu \nu} \nabla_{\nu} \alpha_{0}, \\
\rho_{r}^{\mu \nu}=\eta_{r}^{\mu \nu \alpha \beta} \sigma_{\alpha \beta},
\end{gathered}
$$

where the rank-two tensor coefficients can in general be decomposed in terms of the projection operators $\Xi^{\mu \nu}, b^{\mu} b^{\nu}$, as well as the tensor $b^{\mu \nu}[10]$,

$$
\begin{gathered}
\zeta_{r}^{\mu \nu}=\zeta_{r \perp} \Xi^{\mu \nu}-\zeta_{r \|} b^{\mu} b^{\nu}-\zeta_{r \times} b^{\mu \nu}, \\
\kappa_{r}^{\mu \nu}=\kappa_{r \perp} \Xi^{\mu \nu}-\kappa_{r \|} b^{\mu} b^{\nu}-\kappa_{r \times} b^{\mu \nu},
\end{gathered}
$$

while the rank-four tensor coefficient involves the projection operator $\Delta^{\mu \nu \alpha \beta}$ and products of $\Delta^{\mu \nu}, \Xi^{\mu \nu}, b^{\mu} b^{\nu}$, as well as $b^{\mu \nu}$ (for more details, see Ref. [10]),

$$
\begin{aligned}
\eta_{r}^{\mu \nu \alpha \beta}= & 2 \eta_{r 0} \Delta^{\mu \nu \alpha \beta}+\eta_{r 1}\left(\Delta^{\mu \nu}-\frac{3}{2} \Xi^{\mu \nu}\right)\left(\Delta^{\alpha \beta}-\frac{3}{2} \Xi^{\alpha \beta}\right) \\
& -2 \eta_{r 2}\left(\Xi^{\mu \alpha} b^{\nu} b^{\beta}+\Xi^{\nu \alpha} b^{\mu} b^{\beta}\right) \\
& -2 \eta_{r 3}\left(\Xi^{\mu \alpha} b^{\nu \beta}+\Xi^{\nu \alpha} b^{\mu \beta}\right) \\
& +2 \eta_{r 4}\left(b^{\mu \alpha} b^{\nu} b^{\beta}+b^{\nu \alpha} b^{\mu} b^{\beta}\right) .
\end{aligned}
$$

The scalar transport coefficients $\zeta_{r \perp}, \zeta_{r \|}, \zeta_{r \times}, \kappa_{r \perp}, \kappa_{r \|}, \kappa_{r \times}$, $\eta_{r 0}, \eta_{r 1}, \eta_{r 2}, \eta_{r 3}, \eta_{r 4}$ are obtained by substituting Eqs. (90)(92) into Eqs. (87)-(89) and identifying the coefficients of the corresponding tensor structures.

The bulk-viscosity coefficients $\zeta_{r \perp}, \zeta_{r \|}, \zeta_{r \times}$ are then determined by the following equations:

$$
\begin{aligned}
\sum_{n=0, \neq 1,2}^{N_{0}} \mathcal{A}_{r n}^{(0)} \zeta_{n \perp} & =\alpha_{r}^{(0)}, \\
\sum_{n=0, \neq 1,2}^{N_{0}} \mathcal{A}_{r n}^{(0)}\left(\zeta_{n \perp}-\zeta_{n \|}\right) & =0, \\
\sum_{n=0, \neq 1,2}^{N_{0}} \mathcal{A}_{r n}^{(0)} \zeta_{n \times} & =0
\end{aligned}
$$


and hence in the 14-moment approximation $\left(N_{0}=2\right)$,

$$
\zeta_{0 \perp}=\zeta_{0 \|}=\frac{\alpha_{r}^{(0)}}{\mathcal{A}_{r 0}^{(0)}}, \quad \zeta_{0 \times}=0 .
$$

Note that, as long as the collision integral is assumed to be independent of the magnetic field, only a tensor structure of the type $\sim \Xi^{\mu \nu}-b^{\mu} b^{\nu} \equiv \Delta^{\mu \nu}$ survives in the bulk-viscosity tensor (93). In general, however, this does not need to be the case. An explicit example is given in Ref. [39] where $\zeta_{0 \perp}$ and $\zeta_{0 \|}$ are calculated for a hot quark-gluon plasma in a magnetic field, taking into account Landau quantization.

The transport coefficients $\kappa_{r \perp}, \kappa_{r \|}, \kappa_{r \times}$ are found by inserting Eq. (91) into Eq. (88). This leads to the following system of coupled equations:

$$
\begin{gathered}
\sum_{n=0, \neq 1}^{N_{1}}\left[\mathcal{A}_{r n}^{(1)} \kappa_{n \perp}+\mathfrak{q} B\left(\mathcal{F}_{1-r, n}^{(1)}+\alpha_{r}^{h} \delta_{n 0}\right) \kappa_{n \times}\right]=\alpha_{r}^{(1)}, \\
\sum_{n=0, \neq 1}^{N_{1}} \mathcal{A}_{r n}^{(1)} \kappa_{n \|}=\alpha_{r}^{(1)}, \\
\sum_{n=0, \neq 1}^{N_{1}}\left[\mathcal{A}_{r n}^{(1)} \kappa_{n \times}-\mathfrak{q} B\left(\mathcal{F}_{1-r, n}^{(1)}+\alpha_{r}^{h} \delta_{n 0}\right) \kappa_{n \perp}\right]=0,
\end{gathered}
$$

and hence, in the 14-moment approximation $\left(N_{1}=1\right)$,

$$
\begin{aligned}
\kappa_{r} \equiv \kappa_{0 \|} & =\frac{\alpha_{r}^{(1)}}{\mathcal{A}_{r 0}^{(1)}}, \quad \kappa_{0 \perp}=\kappa_{0 \|}\left[1+\left(\mathfrak{q} B \frac{\mathcal{F}_{1-r, \quad 0}^{(1)}+\alpha_{r}^{h}}{\mathcal{A}_{r 0}^{(1)}}\right)^{2}\right]^{-1}, \\
\kappa_{0 \times} & =\kappa_{0 \perp} \mathfrak{q} B \frac{\mathcal{F}_{1-r, 0}^{(1)}+\alpha_{r}^{h}}{\mathcal{A}_{r 0}^{(1)}} .
\end{aligned}
$$

One observes that, when $B \rightarrow 0, \quad \kappa_{0 \times} \rightarrow 0$, while $\kappa_{0 \|} \rightarrow \kappa_{0 \perp}$. Also in this case, the diffusion tensor $\kappa_{0}^{\mu \nu} \sim \Delta^{\mu \nu}$, as expected. Moreover, for any $B \neq 0$, $\kappa_{0 \perp}<\kappa_{0 \|}$, i.e., due to the cyclotron motion of the particles, particle (or charge) diffusion transverse to the magnetic field is reduced as compared to the diffusion parallel to the magnetic field.

In the limit of a massless Boltzmann gas, where $J_{n q} \equiv I_{n q}=\frac{(n+1) !}{2(2 q+1) ! !} \beta_{0}^{2-n} P_{0}$, and for a constant binary cross section $\sigma=$ const, we obtain for $r=0$ the following expressions: $\quad \alpha_{0}^{(1)}=\beta_{0} P_{0} / 12, \quad \alpha_{0}^{h} \equiv-1 / h_{0}=-\beta_{0} / 4$, $\mathcal{F}_{10}^{(1)}=2 \beta_{0} / 3$, and $\mathcal{A}_{00}^{(1)}=4 /\left(9 \lambda_{\mathrm{mfp}}\right)$, where $\lambda_{\text {mfp }}=$ $1 /\left(n_{f 0} \sigma\right)$ is the mean free path of the particles, and thus the diffusion coefficients assume the values

$$
\begin{aligned}
\kappa_{0 \|} & =\frac{3 \lambda_{\mathrm{mfp}} n_{f 0}}{16}, \quad \kappa_{0 \perp}=\frac{48 \lambda_{\mathrm{mfp}} n_{f 0}}{256+225 \xi_{B}^{2}}, \\
\kappa_{0 \times} & =\frac{45 \xi_{B} \lambda_{\mathrm{mfp}} n_{f 0}}{256+225 \xi_{B}^{2}},
\end{aligned}
$$

where $\xi_{B} \equiv \mathfrak{q} B \beta_{0} \lambda_{\mathrm{mfp}} \equiv \lambda_{\mathrm{mfp}} / R_{T}$ was defined in the Introduction.

As expected, the longitudinal diffusion is solely given in terms of the mean free path, since the magnetic field does not affect the dynamics in the $b^{\mu}$ direction. On the other hand, there is an interplay between the mean free path and the thermal Larmor radius $R_{T}$ for the transverse diffusion, since the underlying particles not only collide but also undergo cyclotron motion. The magnetic-field dependence of these coefficients is shown in Fig. 1(a).

Let us consider the limiting case where the mean free path is much larger than the thermal Larmor radius, i.e., $\xi_{B} \gg 1$. This can be achieved either for fixed $B$ by decreasing the temperature or density, such that the mean free path increases, or by increasing the magnetic field $B$, and thus decreasing the Larmor radius, for fixed density, i.e., fixed mean free path. In this limit,
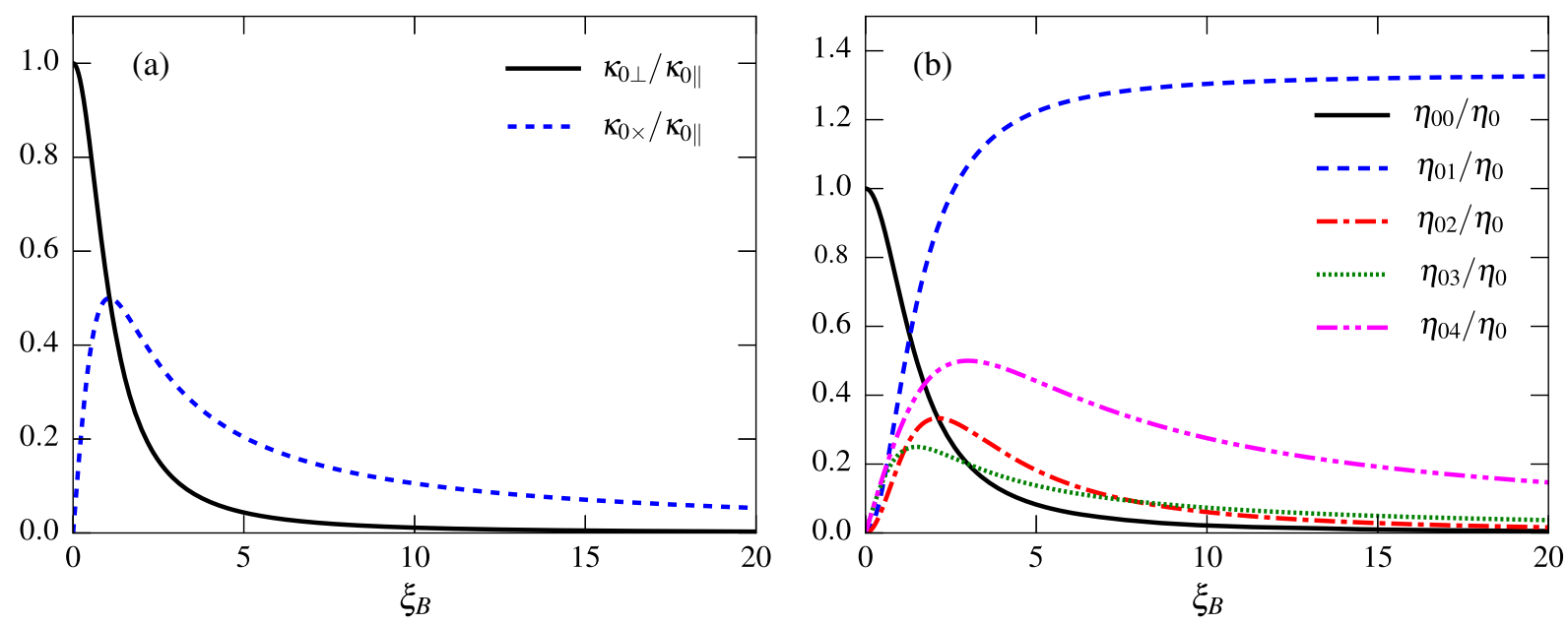

FIG. 1. The magnetic-field dependence of the diffusion coefficients (a) and the shear-viscosity coefficients (b). 


$$
\begin{aligned}
& \kappa_{0 \|}=\frac{3 \lambda_{\mathrm{mfp}} n_{f 0}}{16}, \quad \kappa_{0 \perp} \simeq \frac{16}{75} \frac{\lambda_{\mathrm{mfp}} n_{f 0}}{\xi_{B}^{2}}, \\
& \kappa_{0 \times} \simeq \frac{\lambda_{\mathrm{mfp}} n_{f 0}}{5 \xi_{B}} \equiv \frac{n_{f 0} R_{T}}{5} .
\end{aligned}
$$

As expected, the Hall diffusion coefficient $\kappa_{0 \times}$ assumes a value which is independent of the mean free path. Note, however, that we obtain a nonzero value for this quantity. The unique relationship between the diffusion coefficient and the electric conductivity (the Wiedemann-Franz law mentioned above) then implies that the Hall conductivity is also nonzero. This result is different from the vanishing value quoted in Eq. (8.198) of Ref. [25], valid for a mixture of an ultrarelativistic electron gas and a nonrelativistic ion gas.

Finally, inserting Eq. (92) into Eq. (89) leads to the following set of equations for the shear-viscosity coefficients:

$$
\begin{gathered}
\sum_{n=0}^{N_{2}}\left(\mathcal{A}_{r n}^{(\ell)} \eta_{n 0}+4 \mathfrak{q} B \mathcal{F}_{1-r, n}^{(2)} \eta_{n 3}\right)=\alpha_{r}^{(2)} \\
\sum_{n=0}^{N_{2}}\left(\mathcal{A}_{r n}^{(2)} \eta_{n 3}-\mathfrak{q} B \mathcal{F}_{1-r, n}^{(2)} \eta_{n 0}\right)=0 \\
\sum_{n=0}^{N_{2}}\left(\mathcal{A}_{r n}^{(2)} \eta_{n 4}-\mathcal{A}_{r n}^{(2)} \eta_{n 3}-\mathfrak{q} B \mathcal{F}_{1-r, n}^{(2)} \eta_{n 2}\right)=0 \\
\sum_{n=0}^{N_{2}}\left(\mathcal{A}_{r n}^{(2)} \eta_{n 2}+\mathfrak{q} B \mathcal{F}_{1-r, n}^{(2)} \eta_{n 4}-4 \mathfrak{q} B \mathcal{F}_{1-r, n}^{(2)} \eta_{n 3}\right)=0 \\
\sum_{n=0}^{N_{2}}\left(3 \mathcal{A}_{r n}^{(2)} \eta_{n 1}-16 \mathfrak{q} B \mathcal{F}_{1-r, n}^{(2)} \eta_{n 3}\right)=0
\end{gathered}
$$

In the 14-moment approximation $\left(N_{2}=0\right)$ the above set of equations is solved by

$$
\begin{gathered}
\eta_{00}=\eta_{r}\left[1+4\left(\mathfrak{q} B \frac{\mathcal{F}_{1-r, 0}^{(2)}}{\mathcal{A}_{r 0}^{(2)}}\right)^{2}\right]^{-1} \\
\eta_{01}=\frac{16}{3}\left(\mathfrak{q} B \frac{\mathcal{F}_{1-r, 0}^{(2)}}{\mathcal{A}_{r 0}^{(2)}}\right)^{2} \eta_{00}, \\
\eta_{02}=3\left(\mathfrak{q} B \frac{\mathcal{F}_{1-r, 0}^{(2)}}{\mathcal{A}_{r 0}^{(2)}}\right)^{2}\left[1+\left(\mathfrak{q} B \frac{\mathcal{F}_{1-r, 0}^{(2)}}{\mathcal{A}_{r 0}^{(2)}}\right)^{2}\right]^{-1} \eta_{00}, \\
\eta_{03}=\mathfrak{q} B \frac{\mathcal{F}_{1-r, 0}^{(2)} \eta_{00},}{\mathcal{A}_{r 0}^{(2)}}{ }^{\mathcal{F}_{1-r, 0}^{(2)}}\left[1+\left(\mathfrak{q} B \frac{\mathcal{F}_{1-r, 0}^{(2)}}{\mathcal{A}_{r 0}^{(2)}}\right)^{2}\right]^{-1},
\end{gathered}
$$

where $\eta_{r}=\alpha_{r}^{(2)} / \mathcal{A}_{r 0}^{(2)}$ corresponds to the usual shear-viscosity coefficient. As expected, when $B \rightarrow 0$, only $\eta_{00}$ remains nonzero, such that $\eta_{0}^{\mu \nu \alpha \beta} \sim \Delta^{\mu \nu \alpha \beta}$, as expected. Note that, for $B \neq 0$, the "standard" shear-viscosity coefficient $\eta_{00}$ is reduced as compared to its value for $B=0$. This reduction of viscosity is similar to the mechanism suggested in Ref. [40], giving rise to the so-called "anomalous viscosity," although that work considered gluon instead of electromagnetic fields.

In the limit of a massless Boltzmann gas and for a constant cross section, we obtain for $r=0$ the quantities $\alpha_{0}^{(2)}=4 P_{0} / 5, \mathcal{F}_{10}^{(2)}=\beta_{0} / 5$, and $\mathcal{A}_{00}^{(2)}=3 /\left(5 \lambda_{\text {mfp }}\right)$. This yields $\eta_{0}=4 \lambda_{\mathrm{mfp}} P_{0} / 3$ and

$$
\begin{aligned}
\eta_{00} & =\frac{12 \lambda_{\mathrm{mfp}} P_{0}}{9+4 \xi_{B}^{2}}, \quad \eta_{01}=\frac{64}{9} \frac{\xi_{B}^{2} \lambda_{\mathrm{mfp}} P_{0}}{9+4 \xi_{B}^{2}}, \\
\eta_{02} & =\frac{36 \xi_{B}^{2} \lambda_{\mathrm{mfp}} P_{0}}{\left[9+4 \xi_{B}^{2}\right]\left[9+\xi_{B}^{2}\right]}, \quad \eta_{03}=\frac{4 \xi_{B} \lambda_{\mathrm{mfp}} P_{0}}{9+4 \xi_{B}^{2}} \\
\eta_{04} & =\frac{4 \xi_{B} \lambda_{\mathrm{mfp}} P_{0}}{9+\xi_{B}^{2}} .
\end{aligned}
$$

The magnetic field dependence of these coefficients is shown in Fig. 1(b). For a large ratio of mean free path to thermal Larmor radius, $\xi_{B} \gg 1$,

$$
\begin{aligned}
& \eta_{00}=\frac{1}{3} \eta_{02} \simeq \frac{9}{4} \frac{\eta_{0}}{\xi_{B}^{2}}, \quad \eta_{01} \simeq \frac{4}{3} \eta_{0}, \\
& \eta_{03}=\frac{1}{4} \eta_{04} \simeq \frac{\lambda_{\mathrm{mfp}} P_{0}}{\xi_{B}} \equiv P_{0} R_{T} .
\end{aligned}
$$

In this limit, the last two viscosities, $\eta_{03}$ and $\eta_{04}$, become independent of $\lambda_{\mathrm{mfp}}$. They appear purely due to the Lorentz force (and are thus named Hall viscosities). The relation $\eta_{03}=\eta_{04} / 4$ holds also in the nonrelativistic case [41]. We note that a similar study of the shear-viscosity coefficients in the Navier-Stokes limit was recently performed in Ref. [42], using the Boltzmann equation in the relaxationtime approximation.

Finally, we remark that the effect of a magnetic field on the shear viscosity of a strongly coupled $\mathcal{N}=4$ supersymmetric Yang-Mills plasma with a large number of colors was studied in Ref. [11]. In this case, it was shown that the ratio between $\eta_{00}$ and the entropy density $s$ does not change with the magnetic field, $\eta_{00} / s=1 /(4 \pi)$, while the ratio $\left(\eta_{00}+\eta_{02}\right) / s$, considered in Ref. [11], was found to be suppressed in strong magnetic fields. This illustrates how the microscopic assumptions regarding the fluid, i.e., strong versus weak coupling, may alter its response to magnetic fields. 


\section{Second-order magnetohydrodynamical equations of motion}

We now derive the equations of motion for nonresistive, second-order dissipative magnetohydrodynamics. In this case, all terms in Eqs. (75)-(77) are kept, but the irreducible moments $\rho_{r}^{\mu_{1} \cdots \mu_{\ell}}$ with $r \neq 0$ are replaced using the 14moment approximation $\left(N_{0}=2, N_{1}=1, N_{2}=0\right)$ using Eq. (85). With the definitions (62)-(65) we obtain

$$
\begin{gathered}
\rho_{r}=\sum_{n=0, \neq 1,2}^{N_{0}} \rho_{n} \mathcal{F}_{-r, n}^{(0)} \\
=-\frac{3}{m_{0}^{2}} \Pi \frac{J_{r 0} D_{30}+J_{r+1,0} G_{23}+J_{r+2,0} D_{20}}{J_{20} D_{20}+J_{30} G_{12}+J_{40} D_{10}}, \\
\rho_{r}^{\mu}=\sum_{n=0, \neq 1}^{N_{1}} \rho_{n}^{\mu} \mathcal{F}_{-r, n}^{(1)}=V_{f}^{\mu} \frac{J_{r+2,1} J_{41}-J_{r+3,1} J_{31}}{D_{31}}, \\
\rho_{r}^{\mu \nu}=\sum_{n=0}^{N_{2}} \rho_{n}^{\mu \nu} \mathcal{F}_{-r, n}^{(2)}=\pi^{\mu \nu} \frac{J_{r+4,2}}{J_{42}},
\end{gathered}
$$

while all higher-rank tensors $(\ell>2)$ are assumed to vanish. The above formulas also hold for negative values of $r$.

For $r=0$ Eq. (75), together with Eqs. (115)-(117), leads to an equation of motion for the bulk viscous pressure

$$
\begin{aligned}
\tau_{\Pi} \dot{\Pi}+\Pi= & -\zeta \theta-\ell_{\Pi V} \nabla_{\mu} V_{f}^{\mu}-\tau_{\Pi V} V_{f}^{\mu} \dot{u}_{\mu}-\delta_{\Pi \Pi} \Pi \theta \\
& -\lambda_{\Pi V} V_{f}^{\mu} \nabla_{\mu} \alpha_{0}+\lambda_{\Pi \pi} \pi^{\mu \nu} \sigma_{\mu \nu} .
\end{aligned}
$$

Similarly, taking $r=0$ we obtain a relaxation equation for the particle diffusion current from Eq. (76)

$$
\begin{aligned}
\tau_{V} \dot{V}_{f}^{\langle\mu\rangle}+V_{f}^{\mu}= & \kappa \nabla^{\mu} \alpha_{0}-\tau_{V} V_{f, \nu} \omega^{\nu \mu}-\delta_{V V} V_{f}^{\mu} \theta-\ell_{V \Pi} \nabla^{\mu} \Pi \\
& +\ell_{V \pi} \Delta^{\mu \nu} \nabla_{\lambda} \pi_{\nu}^{\lambda}+\tau_{V \Pi} \Pi \dot{u}^{\mu}-\tau_{V \pi} \pi^{\mu \nu} \dot{u}_{\nu} \\
& -\lambda_{V V} V_{f, \nu} \sigma^{\mu \nu}+\lambda_{V \Pi} \Pi \nabla^{\mu} \alpha_{0}-\lambda_{V \pi} \pi^{\mu \nu} \nabla_{\nu} \alpha_{0} \\
& -\delta_{V B} \mathfrak{q} B b^{\mu \nu} V_{f, \nu} .
\end{aligned}
$$

The relaxation equation of the shear-stress tensor follows from Eq. (77) for $r=0$,

$$
\begin{aligned}
\tau_{\pi} \dot{\pi}^{\langle\mu \nu\rangle}+\pi^{\mu \nu}= & 2 \eta \sigma^{\mu \nu}+2 \tau_{\pi} \pi_{\lambda}^{\langle\mu} \omega^{\nu\rangle \lambda}-\delta_{\pi \pi} \pi^{\mu \nu} \theta \\
& -\tau_{\pi \pi} \pi^{\lambda\langle\mu} \sigma_{\lambda}^{\nu\rangle}+\lambda_{\pi \Pi} \Pi \sigma^{\mu \nu} \\
& -\tau_{\pi V} V_{f}^{\langle\mu} \dot{u}^{\nu\rangle}+\ell_{\pi V} \nabla^{\langle\mu} V_{f}^{\nu\rangle} \\
& +\lambda_{\pi V} V_{f}^{\langle\mu} \nabla^{\nu\rangle} \alpha_{0}-\delta_{\pi B} \mathfrak{q} B b^{\alpha \beta} \Delta_{\alpha \kappa}^{\mu \nu} g_{\lambda \beta} \pi^{\kappa \lambda} .
\end{aligned}
$$

The coefficients of the terms without explicit dependence on the magnetic field are given in Appendix C of Ref. [22] (note that $n^{\mu} \leftrightarrow V_{f}^{\mu}$ and the index $n \leftrightarrow V$ ). In deriving these equations of motion only the linear contributions arising from the collision integrals were retained. We remark that, given our assumptions, the omitted nonlinear terms display no dependence on the magnetic field and were already calculated in Ref. [43].

To the best of our knowledge, Eqs. (118)-(120) provide the first formulation of nonresistive, second-order dissipative magnetohydrodynamics that can be causal and linearly stable around equilibrium, in contrast to the Navier-Stokes approximation derived in Sec. IV B. As such, this new system of equations is suitable to investigate the effects of magnetic fields on relativistic dissipative fluid dynamics, e.g., in heavy-ion collisions.

The coefficient of the term involving the magnetic field in Eq. (119) is

$$
\delta_{V B}=\frac{\mathcal{F}_{10}^{(1)}+\alpha_{0}^{h}}{\mathcal{A}_{00}^{(1)}},
$$

while the corresponding coefficient in Eq. (120) is

$$
\delta_{\pi B}=2 \frac{\mathcal{F}_{10}^{(2)}}{\mathcal{A}_{00}^{(2)}} .
$$

In the limit of a massless Boltzmann gas with constant cross section, $\alpha_{0}^{h}=-\beta_{0} / 4, \mathcal{F}_{10}^{(1)}=2 \beta_{0} / 3, \mathcal{F}_{10}^{(2)}=\beta_{0} / 5$, $\mathcal{A}_{00}^{(1)}=4 /\left(9 \lambda_{\mathrm{mfp}}\right)$, and $\mathcal{A}_{00}^{(2)}=3 /\left(5 \lambda_{\mathrm{mfp}}\right)$, such that

$$
\delta_{V B}=\frac{15}{16} \beta_{0} \lambda_{\mathrm{mfp}}, \quad \delta_{\pi B}=\frac{2}{3} \beta_{0} \lambda_{\mathrm{mfp}} .
$$

Let us finally comment on the first-order Navier-Stokes limit of the second-order equations (118)-(120). Note that the first terms on the right-hand sides, proportional to the standard bulk and shear viscosity as well as particlediffusion coefficients, are actually independent of the magnetic field. But these are not the only first-order terms in these equations: without an assumption about the magnitude of the magnetic field, the last terms in Eqs. (119), (120) are also formally of first order in a small quantity $\left(V_{f, \nu}\right.$ or $\pi^{\kappa \lambda}$, respectively). As demonstrated in Sec. IV B, these terms are to be combined with the firstorder terms on the left-hand side and, after inversion of the respective coefficient matrices, then lead to the various new anisotropic transport coefficients discussed above.

On the other hand, when solving the second-order equations (119) and (120), one does not need to replace the standard viscosity and particle-diffusion coefficients with the new anisotropic transport coefficients found in Sec. IV B, because the effect of the magnetic field is already taken into account by the terms $\sim B$ in these equations. 


\section{CONCLUSIONS AND OUTLOOK}

We have derived, for the first time, the equations of motion for nonresistive, second-order dissipative magnetohydrodynamics from the Boltzmann equation. The derivation is based on the moment expansion of the Boltzmann equation coupled to a magnetic field for a singlecomponent gas of particles without dipole moment or spin. The magnetohydrodynamical equations of motion were obtained in the 14-moment approximation. This is essentially a generalization of Israel-Stewart fluid dynamics to the case of a nonvanishing magnetic field. Despite our simplifying assumptions, the results exhibit the basic structure of second-order dissipative magnetohydrodynamics, in particular how the magnetic field couples to the dynamical evolution of the dissipative quantities. In particular, we note that within our approximations the form of the equations remains close to that of Israel-Stewart theory, with additional terms that couple the fluid to the magnetic field. As such, the new set of second-order dissipative magnetohydrodynamical equations derived here allows one to investigate the effects of magnetic fields in relativistic dissipative fluids in a causal and linearly stable manner. Moreover, we have shown how the first-order transport coefficients split into several components, recovering the results of Refs. $[10,13]$, with the notable difference that there is only one bulk-viscosity coefficient in our approximation. The reason for this is our assumption that the collision integral is independent of the magnetic field.

There are many possible directions for future work. (i) The 14-moment approximation gives only an estimate for the values of the transport coefficients. Improved values can be obtained by resumming higher orders in $N_{\ell}$ in the moment expansion, as demonstrated in Ref. [22]. (ii) Resistive, second-order dissipative magnetohydrodynamics is obtained by keeping the electric field $E^{\mu}$ in the equations of motion. (iii) An extension to spin degrees of freedom allows to include effects of polarization and magnetization [28]. (iv) A relativistic treatment requires to take into account antiparticles with opposite electric charge. These and further questions will be addressed in future work.

\section{ACKNOWLEDGMENTS}

The authors would like to thank T. Lappi for pointing out the similarity of the reduction of dissipative transport coefficients in a magnetic field observed here to the mechanism suggested in Ref. [40]. G. S. D. greatly acknowledges the warm hospitality of the Department of Physics of Goethe University, where part of this work was done. E. M. and D. H. R. greatly acknowledge the warm hospitality of the Department of Physics of the University of Jyväskylä, where part of this work was done. This work was supported by the Collaborative Research Center CRCTR 211 "Strong-interaction matter under extreme conditions" funded by DFG. G. S. D. and J. N. thank Conselho Nacional de Desenvolvimento Científico e Tecnológico (CNPq) for financial support. X. G. H. is supported by the Young 1000 Talents Program of China, NSFC with Grants No. 11535012 and No. 11675041 . E. M. is supported by the Bundesministerium für Bildung und Forschung (BMBF) and by the Research Council of Norway, (NFR) Project No. 255253/F50. H. N. is supported by the European Union's Horizon 2020 research and innovation programme under the Marie Sklodowska-Curie Grant Agreement No. 655285 and by the Academy of Finland, Project No. 297058. J. N. and G. M. M. thank Fundação de Amparo à Pesquisa do Estado de São Paulo (FAPESP) under Grants No. 2015/50266-2 (2017/05685-2) and No. 2016/13517-0, respectively, for financial support. D. H. R. is partially supported by the High-end Foreign Experts Project No. GDW20167100136 of the State Administration of Foreign Experts Affairs of China.

\section{APPENDIX}

Our conventions for the rank-four Levi-Civita tensor $\epsilon^{\mu \nu \alpha \beta}$ are as follows. We take $\epsilon^{0123}=+1$, which implies $\epsilon^{\mu \nu \alpha \beta}=-\epsilon_{\mu \nu \alpha \beta}$. We also have the relations

$$
\begin{aligned}
\epsilon^{\mu \alpha \beta \gamma} \epsilon_{\nu \alpha \rho \sigma}= & \delta_{\nu}^{\mu}\left(\delta_{\sigma}^{\beta} \delta_{\rho}^{\gamma}-\delta_{\rho}^{\beta} \delta_{\sigma}^{\gamma}\right)+\delta_{\rho}^{\mu}\left(\delta_{\nu}^{\beta} \delta_{\sigma}^{\gamma}-\delta_{\sigma}^{\beta} \delta_{\nu}^{\gamma}\right) \\
& +\delta_{\sigma}^{\mu}\left(\delta_{\rho}^{\beta} \delta_{\nu}^{\gamma}-\delta_{\nu}^{\beta} \delta_{\rho}^{\gamma}\right),
\end{aligned}
$$

and

$$
\epsilon^{\mu \nu \alpha \beta} \epsilon_{\kappa \lambda \alpha \beta}=2\left(\delta_{\lambda}^{\mu} \delta_{\kappa}^{\nu}-\delta_{\kappa}^{\mu} \delta_{\lambda}^{\nu}\right) .
$$

In flat Minkowski space, all Kronecker deltas can be replaced by the mixed contra- and covariant metric tensor, e.g., $\delta_{\nu}^{\mu} \equiv g_{\nu}^{\mu}$. 
[1] U. Heinz and R. Snellings, Annu. Rev. Nucl. Part. Sci. 63, 123 (2013).

[2] V. Skokov, A. Y. Illarionov, and V. Toneev, Int. J. Mod. Phys. A 24, 5925 (2009).

[3] W. T. Deng and X. G. Huang, Phys. Rev. C 85, 044907 (2012).

[4] K. Tuchin, Phys. Rev. C 88, 024911 (2013).

[5] A. Bzdak and V. Skokov, Phys. Lett. B 710, 171 (2012).

[6] S. R. de Groot and L. G. Suttorp, Foundations of Electrodynamics (North-Holland, Amsterdam, 1972).

[7] L. Rezzolla and O. Zanotti, Relativistic Hydrodynamics (Oxford University, New York, 2013).

[8] X.-G. Huang, Rep. Prog. Phys. 79, 076302 (2016).

[9] X.-G. Huang, M. Huang, D. H. Rischke, and A. Sedrakian, Phys. Rev. D 81, 045015 (2010).

[10] X.-G. Huang, A. Sedrakian, and D. H. Rischke, Ann. Phys. (Amsterdam) 326, 3075 (2011).

[11] R. Critelli, S. I. Finazzo, M. Zaniboni, and J. Noronha, Phys. Rev. D 90, 066006 (2014).

[12] S. I. Finazzo, R. Critelli, R. Rougemont, and J. Noronha, Phys. Rev. D 94, 054020 (2016); 96, 019903(E) (2017).

[13] J. Hernandez and P. Kovtun, J. High Energy Phys. 05 (2017) 001.

[14] G. Pichon, Ann. Inst. Henri Poincaré, Sect. A (N.S.) 2, 21 (1965).

[15] W. A. Hiscock and L. Lindblom, Ann. Phys. (N.Y.) 151, 466 (1983); Phys. Rev. D 31, 725 (1985); 35, 3723 (1987).

[16] G. S. Denicol, T. Kodama, T. Koide, and P. Mota, J. Phys. G 35, 115102 (2008).

[17] S. Pu, T. Koide, and D. H. Rischke, Phys. Rev. D 81, 114039 (2010).

[18] W. Israel, Ann. Phys. (N.Y.) 100, 310 (1976).

[19] J. M. Stewart, Proc. R. Soc. A 357, 59 (1977); W. Israel and J. M. Stewart, Proc. R. Soc. A 365, 43 (1979).

[20] W. Israel and J. M. Stewart, Ann. Phys. (N.Y.) 118, 341 (1979).

[21] G. S. Denicol, T. Koide, and D. H. Rischke, Phys. Rev. Lett. 105, 162501 (2010).
[22] G. S. Denicol, H. Niemi, E. Molnár, and D. H. Rischke, Phys. Rev. D 85, 114047 (2012); 91, 039902(E) (2015).

[23] G. S. Denicol, E. Molnár, H. Niemi, and D. H. Rischke, Eur. Phys. J. A 48, 170 (2012).

[24] S. Li and H. U. Yee, Phys. Rev. D 97, 056024 (2018).

[25] C. Cercignani and G. M. Kremer, The Relativistic Boltzmann Equation: Theory and Applications (Birkhäuser, Basel, 2002).

[26] J. D. Barrow, R. Maartens, and C. G. Tsagas, Phys. Rep. 449, 131 (2007).

[27] J. D. Bekenstein and E. Oron, Phys. Rev. D 18, 1809 (1978).

[28] W. Israel, Gen. Relativ. Gravit. 9, 451 (1978).

[29] C. Eckart, Phys. Rev. 58, 919 (1940).

[30] L. D. Landau and E. M. Lifshitz, Fluid Dynamics, 2nd ed. (Butterworth-Heinemann, Oxford, 1987).

[31] K. Hattori and Y. Yin, Phys. Rev. Lett. 117, 152002 (2016).

[32] M. Gedalin, Phys. Fluids B 3, 1871 (1991).

[33] M. Gedalin and I. Oiberman, Phys. Rev. E 51, 4901 (1995).

[34] E. Molnár, H. Niemi, and D. H. Rischke, Phys. Rev. D 93, 114025 (2016).

[35] M. Alqahtani, M. Nopoush, and M. Strickland, Prog. Part. Nucl. Phys. 101, 204 (2018).

[36] F. Jüttner, Ann. Phys. (N.Y.) 339, 856 (1911); Z. Phys. 47, 542 (1928).

[37] P. Romatschke and M. Strickland, Phys. Rev. D 68, 036004 (2003).

[38] S. R. de Groot, W. A. van Leeuwen, and Ch. G. van Weert, Relativistic Kinetic Theory: Principles and Applications (North Holland, Amsterdam, 1980).

[39] K. Hattori, X.-G. Huang, D. H. Rischke, and D. Satow, Phys. Rev. D 96, 094009 (2017).

[40] M. Asakawa, S. A. Bass, and B. Muller, Phys. Rev. Lett. 96, 252301 (2006).

[41] E. M. Lifshitz and L. P. Pitaevskii, Physical Kinetics, 1 st ed. (Butterworth-Heinemann, Oxford, 1981).

[42] P. Mohanty, A. Dash, and V. Roy, arXiv:1804.01788.

[43] E. Molnár, H. Niemi, G. S. Denicol, and D. H. Rischke, Phys. Rev. D 89, 074010 (2014). 